Nova Southeastern University

Florida

NOVA SOUTHEASTERN

UNIVERSITY

NSUWorks

Marine \& Environmental Sciences Faculty Articles Department of Marine and Environmental Sciences

11-1-1999

\title{
A Near-Surface Microstructure Sensor System Used During TOGA COARE. Part II: Turbulence Measurements
}

\author{
Alexander Soloviev \\ Nova Southeastern University, <<span class="elink">soloviev@nova.edu \\ Roger Lukas \\ University of Hawaii - Manoa \\ Peter Hacker \\ University of Hawaii - Manoa \\ H. Schoeberlein \\ Johns Hopkins University \\ M. Baker \\ Johns Hopkins University \\ See next page for additional authors
}

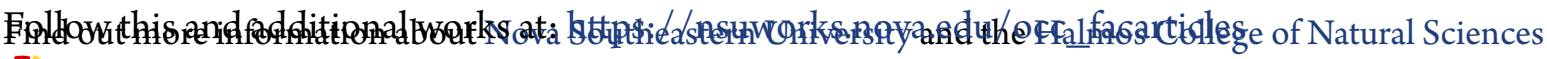

Partofragrepharine Biology Commons, and the Oceanography and Atmospheric Sciences and Meteorology Commons

\section{NSUWorks Citation}

Alexander Soloviev, Roger Lukas, Peter Hacker, H. Schoeberlein, M. Baker, and A. Arjannikov. 1999. A Near-Surface Microstructure Sensor System Used During TOGA COARE. Part II: Turbulence Measurements .Journal of Atmospheric and Oceanic Technology , (11) : 1598 -1618. https://nsuworks.nova.edu/occ_facarticles/633.

This Article is brought to you for free and open access by the Department of Marine and Environmental Sciences at NSUWorks. It has been accepted for inclusion in Marine \& Environmental Sciences Faculty Articles by an authorized administrator of NSUWorks. For more information, please contact nsuworks@nova.edu. 
Authors

A. Arjannikov

Granit - St. Petersburg, Russia

This article is available at NSUWorks: https://nsuworks.nova.edu/occ_facarticles/633 


\title{
A Near-Surface Microstructure Sensor System Used during TOGA COARE. Part II: Turbulence Measurements
}

\author{
A. Soloviev,* R. Lukas, And P. Hacker \\ School of Ocean and Earth Science and Technology, University of Hawaii, Honolulu, Hawaii \\ H. SCHOEBerlein AND M. BAKeR \\ Applied Physics Laboratory, The Johns Hopkins University, Laurel, Maryland
}

A. ArJannikov

Granit, St. Petersburg, Russia

(Manuscript received 24 July 1997, in final form 6 July 1998)

\begin{abstract}
New techniques developed for near-surface turbulence measurements during the Tropical Ocean Global Atmosphere (TOGA) Coupled Ocean-Atmosphere Response Experiment (COARE) employ a difference in spatial scales of turbulence and surface waves. According to this approach, high relative speed of the measurements provides separation of the turbulence and surface wave signals. During the TOGA COARE field studies, highresolution probes of pressure, temperature, conductivity, fluctuation velocity, and acceleration were mounted on the bow of the vessel at a $1.7-\mathrm{m}$ depth in an undisturbed region ahead of the moving vessel. The localization in narrow frequency bands of the vibrations of the bow sensors allows accurate calculation of the dissipation rate. A coherent noise reduction algorithm effectively removes vibration contamination of the velocity dataset. Due to the presence of surface waves and the associated pitching of the vessel, the bow probes "scanned" the near-surface layer of the ocean. Contour plots calculated using the bow signals provide a spatial context for the analysis of near-surface turbulence. A fast-moving free-rising profiler equipped by similar probes sampled the near-surface turbulence during stations. Theory of the three-component electromagnetic velocity sensor and examples of data obtained by bow sensors and free-rising profiler are also presented in this paper.
\end{abstract}

\section{Introduction}

A problem of microstructure and turbulence measurement at the ocean-air interface is the presence of strong surface wave perturbations. They influence the measurements in three different ways. (a) Wave orbital velocities produce a high-frequency fluctuation velocity signal that disturbs the turbulence measurement. This is a linear disturbance that can be removed from the signal using statistical filtering (see, e.g., Agrawal et al. 1992). (b) The orbital velocities modulate the relative flow speed, which results in a frequency modulation of the measured turbulence signal. This is a nonlinear distur-

\footnotetext{
* Permanent affiliation: Oceanography Center, Nova Southeastern University, Dania, Florida.
}

Corresponding author address: Dr. Alexander Soloviev, Oceanography Center, Nova Southeastern University, 8000 North Ocean Dr., Dania, FL 33004.

E-mail: soloviev@ocean.nova.edu bance that is very difficult to remove from the useful signal. (c) The orbital velocities influence the direction of the relative flow, requiring special directional response of the sensors.

For the fluctuation-velocity measurements in the near-surface layer of the ocean all three problems (a)(c) are important, while for the temperature and conductivity measurements only (b) and (c) are relevant. Sensor intersection of the ocean-air interface and areas with air bubbles are another difficulty. This mainly concerns the conductivity signal but may also be a problem for the velocity and temperature measurements.

Microstructure and turbulence measurements in the near-surface layer of the ocean demand special instrumentation and techniques. Requirements for the nearsurface sensor system are different from those for deep ocean measurements. In the latter case, the problem is the low level of small-scale velocity fluctuations; the noise level of the velocity sensor is a crucial parameter. For near-surface measurement, the main problem is the environmental disturbances (surface waves, bubble areas, ship's wake, etc.). The environmental disturbances 
to the useful signal strongly depend on the techniques of measurement. Ultimately, the techniques of measurement impose requirements on the sensor's performance. The sensor's noise level, response time, and spatial resolution are, however, common considerations for both deep-sea and near-surface measurements.

During the Tropical Ocean Global Atmosphere (TOGA) Coupled Ocean-Atmosphere Response Experiment (COARE), our experimental approach was to make microstructure and turbulence measurements in the near-surface layer of the ocean from a fast-moving vehicle using velocity probes with linear output. According to Stewart and Grant (1962) and Soloviev et al. (1988), the high relative speed of measurement provides effective separation of the turbulence and the surface wave signals. The measurements during TOGA COARE were done using bow sensors and a free-rising profiler (FRP). This gave us the ability to solve problems (a)(c).

The bow sensors were originally designed in "Granit" (St. Petersburg, Russia) for microstructure and turbulence measurements within the depth range of 0-500 m (Arjannikov et al. 1979; Turenko 1985). They included the conductivity, temperature, and electromagnetic velocity sensors. For TOGA COARE, a pressure sensor, a U.S. acceleration sensor, and a U.S. data acquisition system were added to the bow measurement system. The bow sensors employed for TOGA COARE are described by Soloviev et al. (1998). In the present paper, we focus on the fluctuation velocity measurements by the bow sensors and the FRP.

\section{Fluctuation velocity measurements from a moving ship}

The electromagnetic velocity and acceleration (EMVA) probe measures the three components of fluctuation velocity vector and the vibration acceleration. The operation of the probe is based on the principle of electromagnetic induction. In contrast to traditional internal-flow electromagnetic sensors, this sensor exploits an external part of the magnetic field. The potential field is formed only by the part of the magnetic field that emerges beyond the limits of the magnetic gap (Arjannikov et al. 1979). As a result, the primary sensor has a perfect hydrodynamic form and a low hydrodynamics noise level. The EMVA probe is the linear device for a wide flow-speed range $\left(0-12.5 \mathrm{~m} \mathrm{~s}^{-1}\right)$. Electrical signals proportional to longitudinal $\left(V_{x}\right)$, transverse $\left(V_{y}\right)$, and vertical $\left(V_{z}\right)$ components of the fluctuation velocity vector appear at the output of the low-frequency amplifiers. The theory of the electromagnetic velocity sensor is described in appendix A.

The noise of the amplifiers dominates the high-frequency range of the sensor (Fig. 1). In the low-frequency part, the electrochemical processes on measuring electrodes are a principal limitation for the electromagnetic velocity sensors with permanent magnets. To reduce

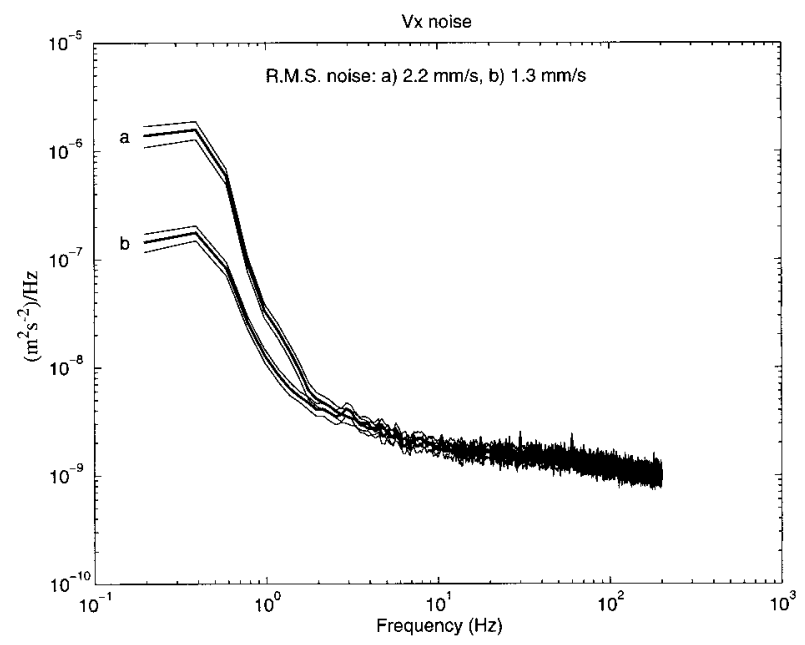

FIG. 1. Noise spectra of the electromagnetic velocity sensor according to laboratory tests at the University of Hawaii (14-15 May 1994): (a) in $\sim 10$ min after placing the sensor with previously dry measuring electrodes to the seawater and (b) $15 \mathrm{~h}$ later. Thin lines represent $95 \%$ confidence intervals. The spectra are calculated from 10-min segments using the MATLAB Signal Processing Toolbox. The 10-min sequences of length 240000 points are divided into 50\% overlapping sections of 2048 points each. Successive sections are detrended, Hanning windowed, transformed with an 2048-point FFT, and averaged. No additional smoothing of spectral components is necessary because of the large number of FFTs (233). The frequency resolution is very fine $(\Delta f=0.1953 \mathrm{~Hz})$

low-frequency polarization noises, the electrodes are made of platinum and the signal is passed through a high-frequency filter with a $0.05-\mathrm{Hz}$ cutoff frequency. After placing the sensor with dry electrodes in seawater, it takes about $24 \mathrm{~h}$ for full equilibration of the electrochemical processes. Figure 1 shows the evolution of the noise spectrum after placing the sensor with previously dry electrodes into the seawater. The rms noise level of the sensor after $15 \mathrm{~h}$ in the seawater reduced from 2.2 to $1.3 \mathrm{~mm} \mathrm{~s}^{-1}$.

Figure 2 demonstrates spectra of all three velocity components $\left(V_{x}, V_{y}\right.$, and $\left.V_{z}\right)$ measured from the bow of the vessel at a $3.2 \mathrm{~m} \mathrm{~s}^{-1}$ ship speed in comparison with the corresponding noise spectra obtained from the postcruise tests. The high-resolution spectra are calculated from 10-min segments similar to those given in Fig. 1. The spectra of all three velocity components are contaminated by the vibration noises at high frequencies. Some of the vibration peaks, presumably associated with the resonant frequencies of ship's body, are relatively narrow. Several other peaks are wider, apparently resulted from the bow frame vibrations. The resonant properties of the bow frame are different from those of the ship because of a different connected water mass, thus resulting in a wider vibration spectrum. The bow frame is not as mechanically rigid in the $y$ direction as in the $x$ and $z$ directions. The $V_{y}$ spectrum has a prominent, relatively broad peak at $\sim 10 \mathrm{~Hz}$ frequency, which is not seen on the corresponding $V_{x}$ and $V_{z}$ spectra. This 

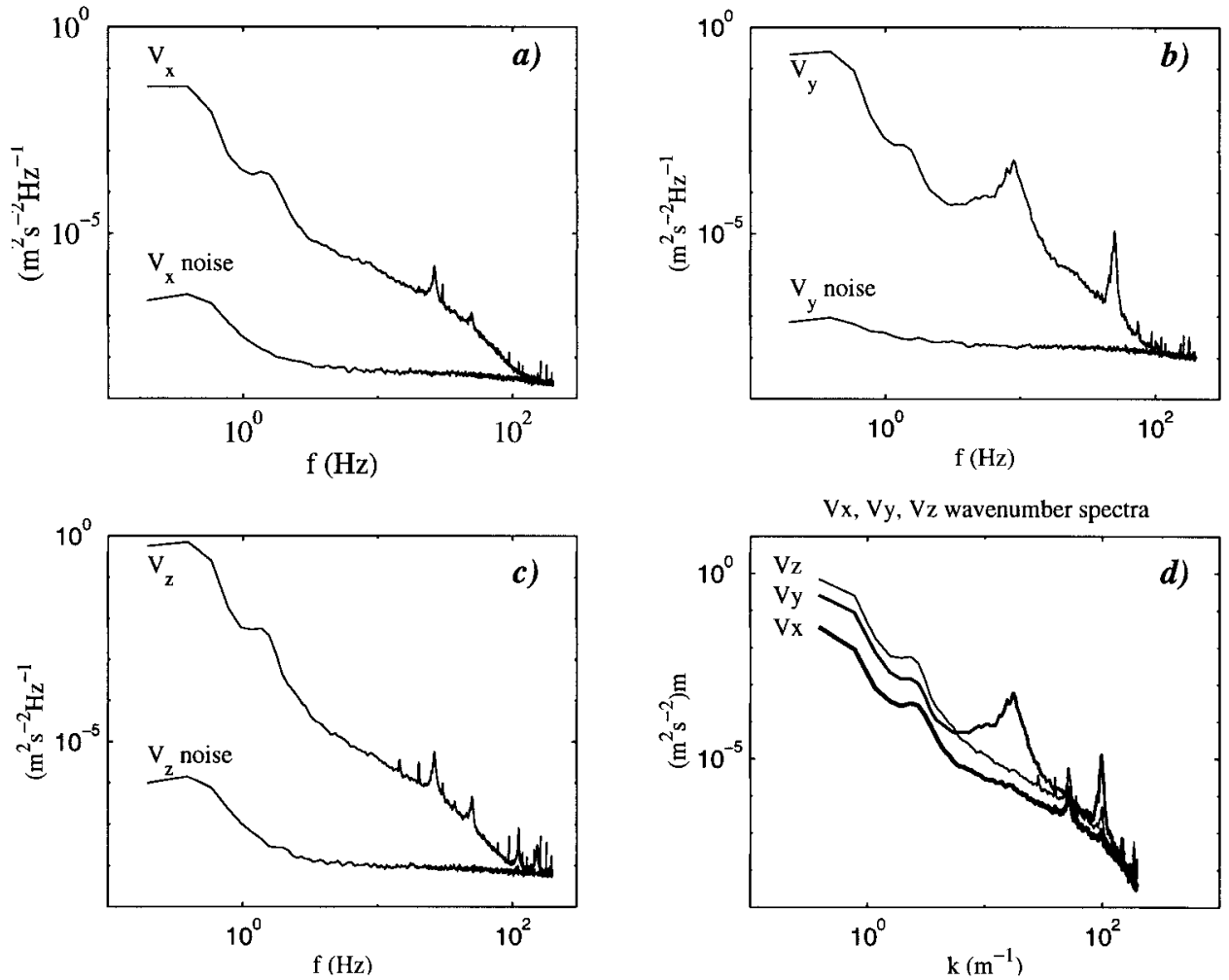

FIG. 2. (a)-(c) Frequency and (d) wavenumber spectra of all three components of velocity vector measured from the R/V Moana Wave on 4 May 1994 at ship speed $3.2 \mathrm{~m} \mathrm{~s}^{-1}$, ship direction $45^{\circ}$, wind speed $4.6 \mathrm{~m}$ $\mathrm{s}^{-1}$, wind direction $75^{\circ}$, swell wave height $1 \mathrm{~m}$, and swell wave direction $50^{\circ}$. Noise spectra are measured in laboratory conditions using the same experimental setup. Wavenumber spectra (d) are corrected for the noise, for the antialias low-pass filter with a $160-\mathrm{Hz}$ cutoff frequency, and for the spatial averaging. Note strong vibration peaks on $V_{y}$ component.

peak is apparently associated with the bow frame vibration in the $y$ direction.

Variation of the vertical component $\left(V_{z}\right)$ is usually larger than that of $V_{x}$ or $V_{y}$ because of the ship pitching. The $V_{z}$ signal was therefore sometimes out of the linear sensor's range. The precruise calibrations for $V_{x}, V_{y}$, and $V_{z}$ channels were done at the Granit testing facility (see Soloviev et al. 1998). The standard calibration procedure at Granit inferred an isotropy of the submerged jet in the calibration water tank. This resulted in an underestimation of the gains for the transverse velocity components $\left(V_{y}\right.$ and $\left.V_{z}\right)$. On scales corresponding to the inertial subrange and outside the vibration peaks, the $V_{y}$ and $V_{z}$ spectra do seem to be somewhat higher than the $V_{x}$ spectrum (Fig. 2d).

The longitudinal $\left(V_{x}\right)$ channel is less contaminated by vibrations and pitching of the ship than the $V_{y}$ and $V_{z}$ channels. Also, the electronics signal-to-noise ratio for the $V_{x}$ channel is about two times better than for the $V_{y}$ and $V_{z}$ channels (see appendix A). The above factors predetermined our choice of the $V_{x}$ channel for the further quantitative analysis of near-surface turbulence. With proper calibration, the transverse channels, $V_{y}$ and
$V_{z}$, can also be useful for calculation of the turbulence characteristics.

Figure 3 shows two examples of the $V_{x}$ velocity spectra at two different ship speeds obtained under low wind speed conditions $\left(U_{a} \sim 4.5 \mathrm{~m} \mathrm{~s}^{-1}\right)$. The time interval between these two tests was 10 min. In Figs. 3a and 3d, the spectrum of $V_{x}$ is compared to the spectrum of noise and to the spectrum of integrated acceleration $g_{x}$ (thin line). A fit of the experimental spectrum (dotted line) by the universal spectrum of turbulence in the intertiaviscous interval is shown in Figs. $3 \mathrm{c}$ and $3 \mathrm{f}$ by solid lines. Vibration contamination, a key issue for the velocity measurements, occurs in narrow frequency bands that do not preclude accurate estimates of the kinetic energy dissipation rate when using a fine-resolution velocity spectrum and the Stewart's and Grant (1962) techniques. The dissipation rate estimates, $\varepsilon$, obtained at the two different ship speeds (Figs. 3c and 3f) differ only by $24 \%$. Note also some difference in the mean depth, $P_{0}$, of the probes and the wind speed between these two tests.

According to Oakey and Elliott (1982), individual estimates of $\varepsilon$ based on assumptions of isotropy are 

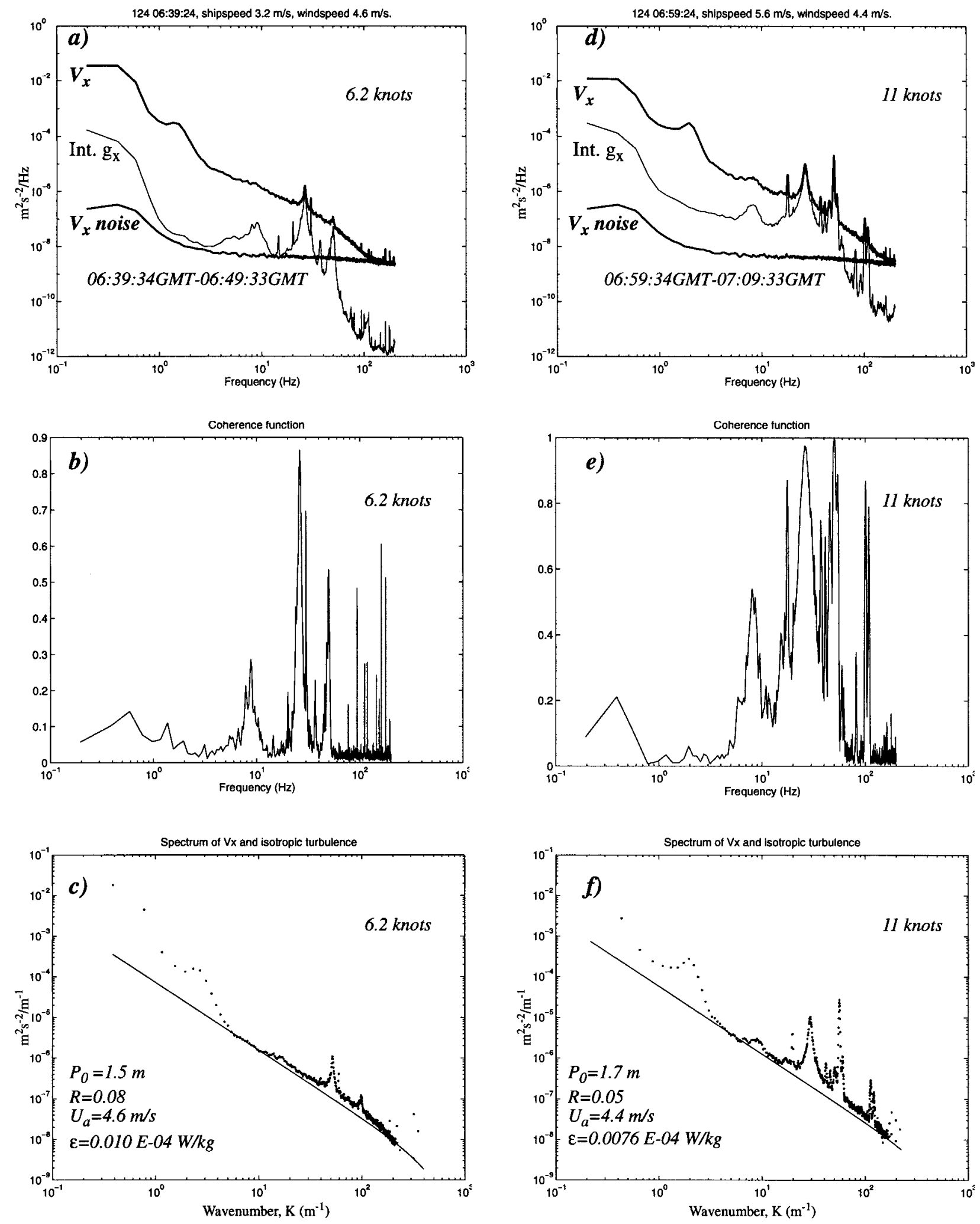

FIG. 3. Tests at two different ship speeds: 6.2 and $11 \mathrm{kt}$ (4 May 1994) (ship direction $45^{\circ}$, wind speed $4.6 \mathrm{~m} \mathrm{~s}^{-1}$, wind direction $75^{\circ}$, swell wave height $\sim 1 \mathrm{~m}$, swell wave direction $\sim 50^{\circ}$ ). 
TABLE 1. Error of dissipation rate estimation at bow measurements.

\begin{tabular}{ccc}
\hline \hline Ship speed $\left(\mathrm{m} \mathrm{s}^{-1}\right)$ & $\begin{array}{c}\text { Electronics noise only } \\
\left(\mathrm{W} \mathrm{kg}^{-1}\right)\end{array}$ & $\begin{array}{c}\text { Electronics noise and } \\
\text { vibrations }\left(\mathrm{W} \mathrm{kg}^{-1}\right)\end{array}$ \\
\hline 3.2 & $1.4 \times 10^{-11}$ & $1.0 \times 10^{-9}$ \\
5.6 & $7.9 \times 10^{-12}$ & $1.7 \times 10^{-9}$ \\
\hline
\end{tabular}

considered to be known within a factor of 2 . The error of the dissipation rate estimate from the bow velocity measurement also depends on the electronic and hydrodynamic noise of velocity sensor and on the vibration contamination.

Table 1 shows the error of dissipation rate estimates for the two examples with different ship speeds shown in Fig. 3. The universal spectrum of Stewart and Grant (1962) was initially fit to the laboratory $V_{x}$ noise spectrum (second column) and then to the sum of the laboratory $V_{x}$ noise spectrum and the integrated $x$-acceleration spectrum (third column).

According to Table 1 (column 2), the influence of the electronics noise for estimating $\varepsilon$ decreases with the increase of ship speed. This is because the ratio of useful (turbulent) signal to electronics noise increases with increase of the ship speed. The length scales of turbulent eddies corresponding to the nominal frequency range of electromagnetic velocity sensor $(0.05-160 \mathrm{~Hz})$ increase proportionally to the ship speed, $U$. The intensity of turbulence in an inertial subrange increases with length scale according to the $5 / 3$ power. Correspondingly, the turbulence signal to electronics noise ratio increases as $\sim U^{5 / 3}$. The combined error (electronics plus vibration noise), however, slightly increases with ship speed (third column of Table 1). The vibration noise apparently decreases with ship speed (see Fig. 3). However, the relative rms variation of the flow, $R$, increases at decreasing ship speed, thus limiting the lower ship speed to $\sim 2 \mathrm{~m}$ $\mathrm{s}^{-1}$ (when $R$ does not exceed a $10 \%$ level and the Taylor hypothesis of frozen turbulence $[f=U k]$ can therefore be used).

The third column in Table 1 actually represents the minimum dissipation rate level that can be estimated from the bow velocity measurement using Stewart's and Grant (1962) techniques. The minimum value of the dissipation rate observed during the 30 days of measurements in the R/V Moana Wave COARE EQ-3 cruise using the bow sensors and the Stewart's and Grant (1962) techniques was equal to $7.510^{-9} \mathrm{~W} \mathrm{~kg}^{-1}$. This is consistent with the values given in the third column of Table 1 .

The coherence estimates shown in Figs. $3 \mathrm{~b}$ and $3 \mathrm{e}$ indicate high coherence between the velocity and acceleration signals only at frequencies where vibration contamination occurs. Based on the high coherence between the velocity and the acceleration signals, the vibration contamination is expected to be reduced significantly by applying coherent noise cancellation techniques. Figure 4 shows an example of the signal before and after applying the coherent noise cancellation al- gorithm developed at The Johns Hopkins University Applied Physics Laboratory (JHU-APL; see appendix B). The algorithm eliminates the fraction of measured spectrum contaminated by vibration without modifying spectrum of ocean velocity. It implements an optimal Wiener filter in time domain. Figure 4 includes the time series before $\left(V_{x}^{\prime}\right)$ and after $\left(v_{x}^{\prime}\right)$ applying the JHU-APL algorithm and the integrated acceleration $g_{x}^{\prime}$. For this example, we selected a 212.5 -s piece of data with all the sensors in the water. Before applying the noise cancellation algorithm the velocity signal is high passed. The high-pass filter in this case is the difference between the original data and a sliding average over 40 points corresponding to a 0.1 -s time window. The choice of this filter is not critical; there are much better filters, and any of these would work well in this application. The time series before and after applying the noise cancellation algorithm is shown in Fig. 4. Using the coherent noise reduction techniques opens the possibility to remove the vibration contamination from the velocity signal and achieve the lower limit of dissipation rate from the moving ship up to $\sim 10^{-11} \mathrm{~W} \mathrm{~kg}^{-1}$ (see the second column in Table 1).

To study spatial effects, the bow velocity data can be analyzed together with the contour plots of temperature, salinity, and density calculated using the variation of the probe's depth due to the vessel pitching (Soloviev and Lukas 1996). An example of measurement taken at intersection of a freshwater lens in the western Pacific warm pool is shown in Fig. 5. Temperature $(T)$ and salinity $(S)$ vary with depth (pressure) variation of the sensors because of the stratification. The density contour plot reveals internal waves in the near-surface layer with amplitude exceeding the pitching amplitude of the vessel. The longitudinal velocity signal processed using the coherent noise cancellation algorithm reveals presence of turbulent spots of $\sim 10-\mathrm{m}$ horizontal scales (Fig. $5 \mathrm{~d}$ ). They are presumably connected with three-dimensional, boundary layer mixing processes. Salinity $S$ versus density $\sigma_{t}$ contour plot (resolving spatial scales not less than $200 \mathrm{~m}$ ) shows no features (Fig. 5f), suggesting that within the freshwater lens the mixing is inhibited on larger spatial scales. The physical analysis of near-surface processes is, however, out of the scope of this paper.

\section{Dissipation rate of turbulent kinetic energy (TKE)}

Figure 6a shows dissipation rate of TKE $(\varepsilon)$ obtained from the bow data using Stewart's and Grant's (1962) techniques. This techniques assumes the Kolmogorov's hypothesis of locally isotropic turbulence for the portion of the velocity spectrum measured. The universal spectrum of turbulence in inertia-viscous interval is fitted from below. When the universal curve touches the experimental spectrum, the corresponding value of $\varepsilon$ rep- 


\section{JHU/APL coherent noise cancellation}
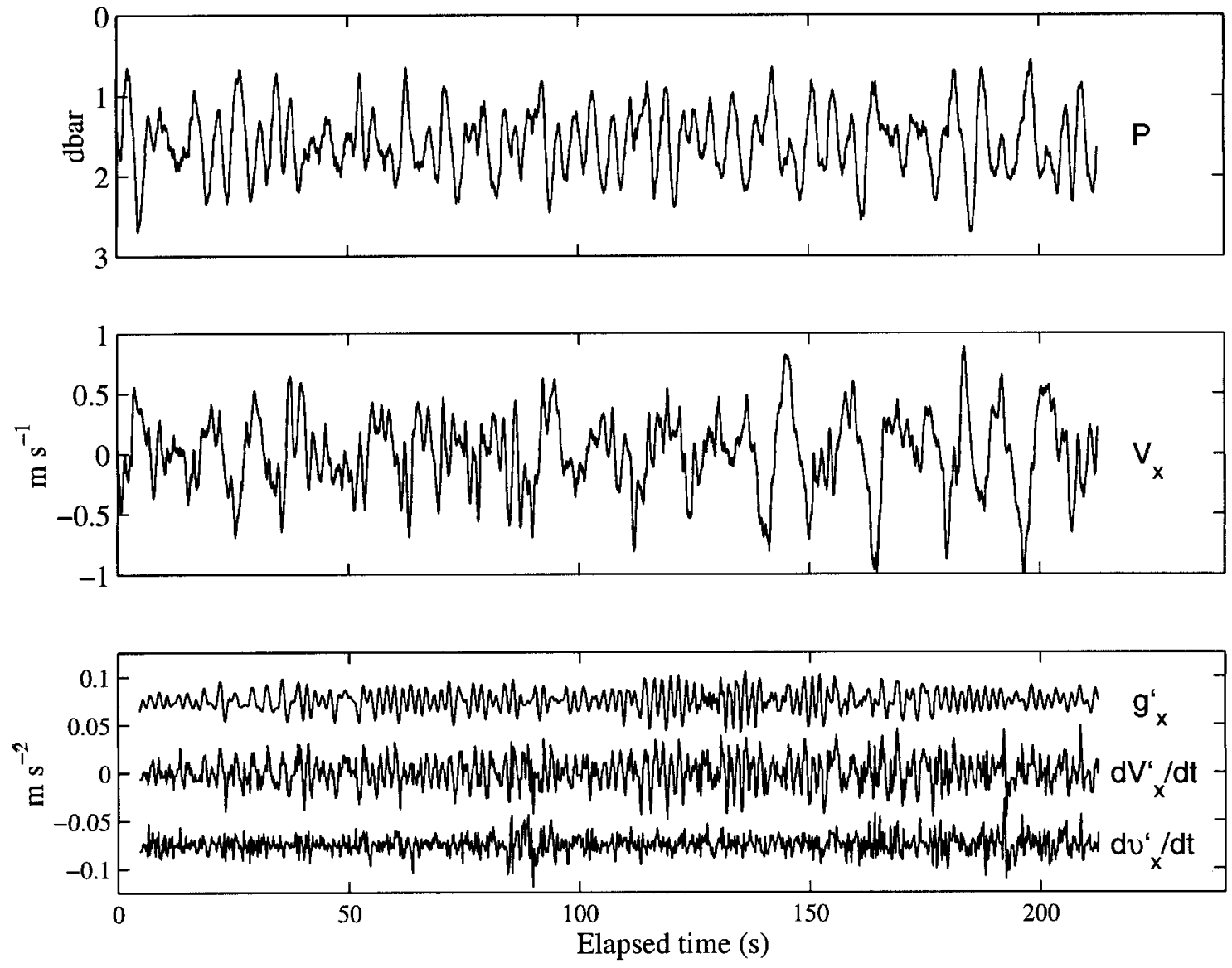

FIG. 4. Example of applying the coherent noise cancellation algorithm to reduce the vibration contamination. Wind speed is variable (squall) $\sim 6 \mathrm{~m} \mathrm{~s}^{-1}$, wind direction $\sim 320^{\circ}$, ship speed $5.4 \mathrm{~m} \mathrm{~s}^{-1}$, ship direction $45^{\circ}$, swell wave height $\sim 1 \mathrm{~m}$, and swell wave direction $\sim 10^{\circ}$. Here $P$ is the pressure (depth), $V_{x}^{\prime}$ is the longitudinal velocity time series before applying the JHU-APL algorithm, and $g_{x}^{\prime}$ is the integrated acceleration. Here $d V_{x}^{\prime} / d t$ and $d v_{x}^{\prime} / d t$ are the longitudinal velocity derivatives before and after applying the JHU-APL algorithm.

resents an upper estimate of the dissipation rate. This is because no disturbances of the kind discussed above (surface waves, ship's motions, etc.) can reduce the measured spectrum. An automatic procedure based on Stewart and Grant's techniques (additionally implying the statistical scatter) has been developed for the dissipation rate estimation.

Values of $\varepsilon$ are calculated from 10-min segments during 4 days of measurements in the $\mathrm{R} / \mathrm{V}$ Moana Wave COARE EQ-3 cruise. We selected these days because of relatively high wind speed conditions $\left(5-16 \mathrm{~m} \mathrm{~s}^{-1}\right)$. The dissipation rates increase with wind speed (Fig. 6a). According to the error considerations described in section 2, the error level of $\varepsilon$ estimation using this technique is $\sim 10^{-9} \mathrm{~W} \mathrm{~kg}^{-1}$. Values of $\varepsilon$ observed in the near-surface layer of the ocean on 7-11 May 1994 (Fig. 6a) greatly exceed this error level.

In Fig. 6b, the field data are compared with theoretical models. Following Soloviev et al. (1988), Agrawal et al. (1992), and Anis and Moum (1995), we use the non- dimensional scaling of the dissipation rate, $\tilde{\varepsilon}=$ $\varepsilon \kappa z / u_{*}^{3}$, and of the depth, $\tilde{z}=g z / u_{*}^{2}$, where $\varepsilon$ is the dissipation rate of the TKE, $\kappa$ is von Kármán's constant, $z$ is the depth, $u_{*}$ is the friction velocity in the water, and $g$ is the acceleration of gravity. The friction velocity was calculated using the TOGA COARE bulk-flux algorithm (Fairall et al. 1996).

The model of the logarithmic boundary layer is helpful for understanding how strongly the near-surface turbulence is enhanced by the surface waves. The logarithmic boundary layer prediction, $\widetilde{\varepsilon}=1$, is shown by the vertical dashed line (Fig. 6b). The model of Craig and Banner (1994), employing the level 21/2 turbulence closure scheme of Mellor and Yamada (1982), gives a parameterization of the wave-enhanced turbulence. The scaling of dissipation rate using $\tilde{\varepsilon}$ and $\tilde{z}$ is compatible with the Craig and Banner (1994) model. The surface roughness length from the water side, $z_{0}$, is a critical parameter of the Craig and Banner (1994) model. We have used a constant value $z_{0}=0.05 \mathrm{~m}$ and a simple 

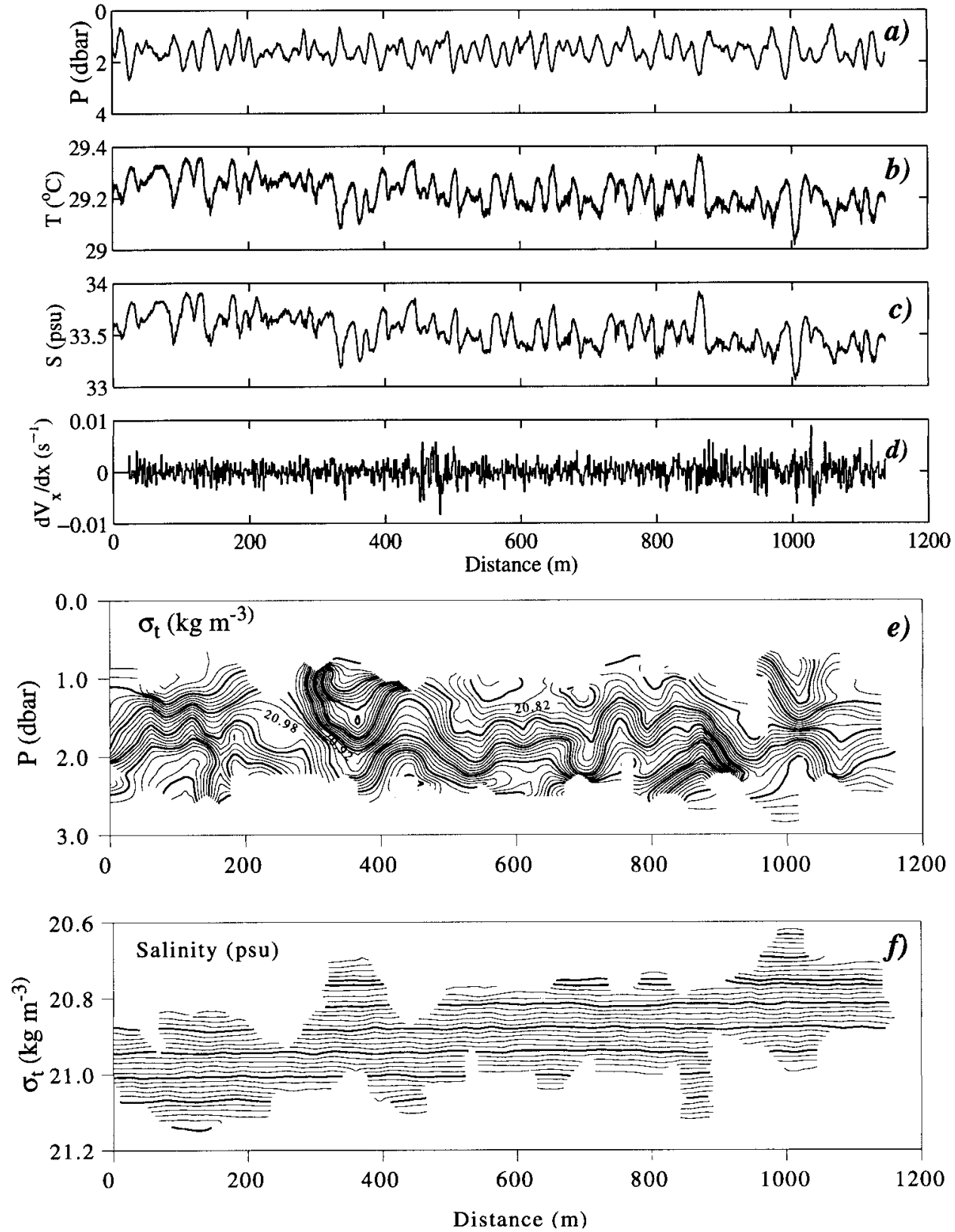

FIG. 5. Example of observations during intersection of a freshwater lens (COARE Moana Wave EQ-3 leg, $2.16^{\circ} \mathrm{N}, 137.02^{\circ} \mathrm{E}$ ). Wind speed is variable (squall) $\sim 6 \mathrm{~m} \mathrm{~s}^{-1}$, wind direction $\sim 320^{\circ}$, ship speed $5.4 \mathrm{~m} \mathrm{~s}^{-1}$, ship direction $45^{\circ}$, swell wave height $\sim 1 \mathrm{~m}$, and swell wave direction $\sim 10^{\circ}$. Variability of the temperature $(T)$ and salinity $(S)$ signals are mainly because of the depth variation of sensors within the stratified nearsurface layer. (d) The $V_{x}$ fluctuation velocity signal is processed using the JHU-APL noise cancellation algorithm. The lower part shows the corresponding contour plots of (e) $\sigma_{t}$ vs $P$ and (f) of $S$ vs $\sigma_{t}$ from measurement by bow sensors.

parameterization (Charnock 1955; Bye 1988) $z_{0}=$ $a u_{*}^{2} / g$, where $a=1400$. The field data are mainly between these two curves.

In Fig. 6b, the Craig and Banner (1994) model is representative of only stationary conditions. The recent study of Smyth et al. (1997) shows that nonstationary effects concerned with heavy rainfall are important in the warm pool area. Note also that the data given in Fig. $6 \mathrm{~b}$ have not been sorted by stratification, wave conditions, etc. The near-surface stratification produced by diurnal warming and rain events may apparently influence the near-surface turbulence. 

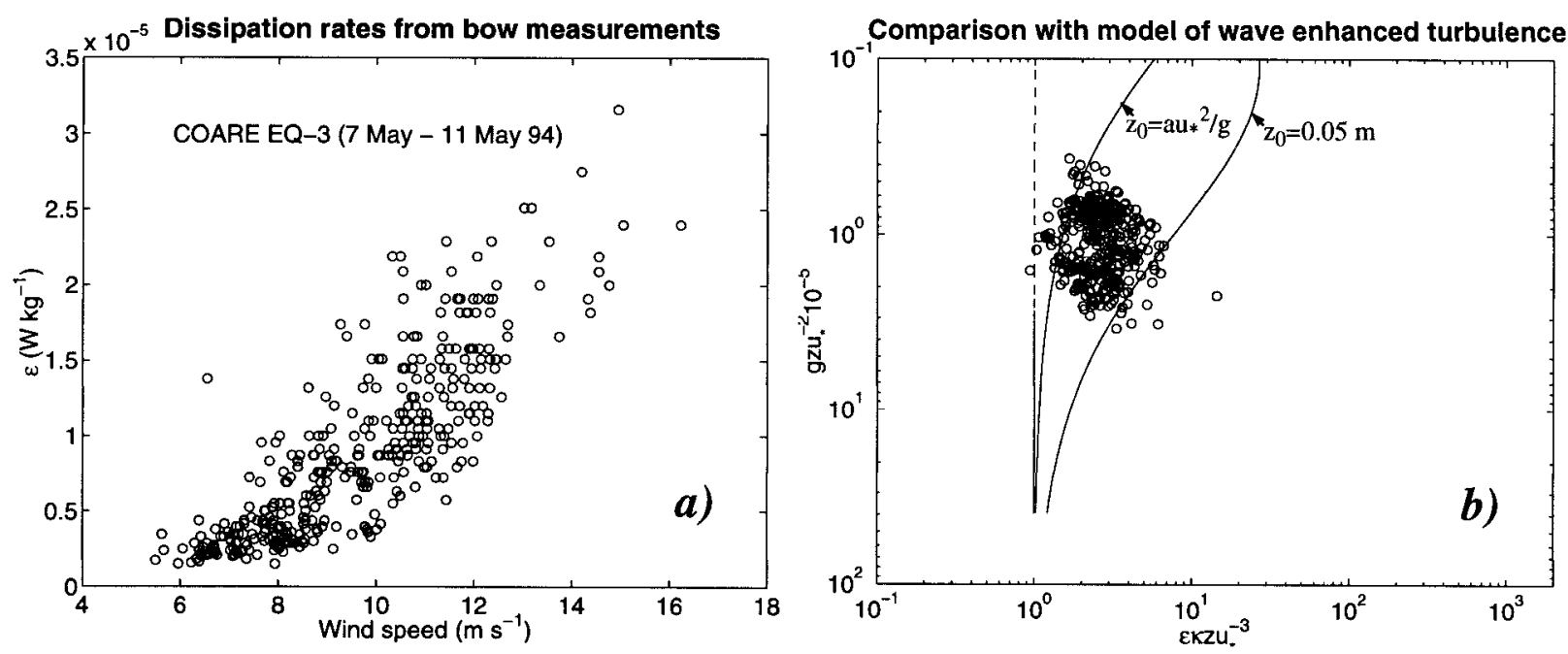

FIG. 6. (a) Dissipation rate of TKE calculated from 10-min bow data segments during 4 days (7-11 May 1994) in the R/V Moana Wave EQ-3 cruise. (b) Same data compared in dimensionless coordinates to logarithmic layer model $\varepsilon=u_{*}^{3} /(\kappa z)$ (vertical dashed line) and Craig and Banner's (1994) model of wave-enhanced turbulence at two different parameterizations of $z_{0}$ (contiguous lines).

\section{Fluctuation velocity measurements using free-rising profiler}

The profiler's body is a hydrodynamic cylinder with a semispherical front constructed from a dense foam, which is positively buoyant (Soloviev et al. 1995). The tail section uses a weighted ring with stabilizers to assure vertical orientation during ascent and to increase the efficiency of water flow around the instrument. The electrical communication cable attaches at the tail section so as not to disturb the water being measured. The three sensors protrude $15 \mathrm{~cm}$ out of the front of the instrument, which also ensures undisturbed water is being sampled by the sensors. The underwater electronics housing fits inside the foam cylinder and is rated to a pressure of $50 \mathrm{db}$. A delivery device is used to shuttle the FRP down to its desired pressure, 15-25 m, where a pressure sensitive mechanism releases the FRP for its ascent to the surface.

The measurements with the free-rising profiler were made from the drifting ship. The profiler connected with the "shuttle" (winged frame) was deployed from the stern of the R/V Moana Wave with the help of a metal frame, which allowed the device to slide from the ship to the water. After leaving the metal frame, the profiler fell into the water and slid outside the ship's wake a distance of about 15-35 m from ship's wake as it sank. This distance depended on the drift of the ship and the intensity of near-surface currents. At the desired depth the pressure release mechanism released the profiler from the shuttle and the profiler turned to a vertical orientation. The profiler then ascended to the surface from the depth of detachment of the shuttle with a vertical velocity of 2-3 $\mathrm{m} \mathrm{s}^{-1}$. The ascent rate depended on the net buoyancy of the profiler. The profiler had a rather large net buoyancy-to-weight ratio that provided nearly constant vertical speed of the profiler with respect to the surrounding water mass [see details in Soloviev et al. (1988)]. The technical characteristics of the FRP probes are given in Table 2. A more detailed description is in Soloviev et al. (1995).

An example of measurement using the FRP is shown in Fig. 7. The mean ascent rate of the profiler during this measurement was $2.9 \mathrm{~m} \mathrm{~s}^{-1}$. The vertical salinity profile shows a salinity depression within the upper 5 $\mathrm{m}$ because of a previous rain. Subsequent diurnal warming develops within the freshwater lens, additionally increasing its stability. Turbulent mixing is also mainly localized within this stably stratified near-surface layer.

TABLE 2. Main technical characteristics of fluctuation velocity probes mounted on the bow of the vessel and on the free-rising profiler during the R/V Moana Wave COARE MW9410 and MW9411 legs.

\begin{tabular}{lcccc}
\hline \hline \multicolumn{1}{c}{ Parameter } & Bow sensor $\left(V_{x}\right)$ & Bow sensor $\left(V_{y}\right)$ & Bow sensor $\left(V_{z}\right)$ & Free-rising profiler $\left(V_{x}\right) *$ \\
\hline Range $\left(\mathrm{m} \mathrm{s}^{-1}\right)$ & \pm 2.5 & \pm 5 & \pm 5 & \pm 0.25 \\
Accuracy $(\%)$ & $<5$ & $<5$ & $<5$ & $<5$ \\
Noise level $\left(\mathrm{mm} \mathrm{s}^{-1}\right)^{* *}$ & 1 & 2 & 2 & $<1$ \\
Frequency range $(\mathrm{Hz})$ & $0.05-200$ & $0.05-200$ & $0.05-200$ & $2-150$ \\
Digital resolution $\left(\mathrm{mm} \mathrm{s}^{-1}\right)$ & 1.1 & 2.2 & 2.2 & 0.6 \\
\hline
\end{tabular}

* One component velocity sensor.

** After $24 \mathrm{~h}$ in saltwater. 

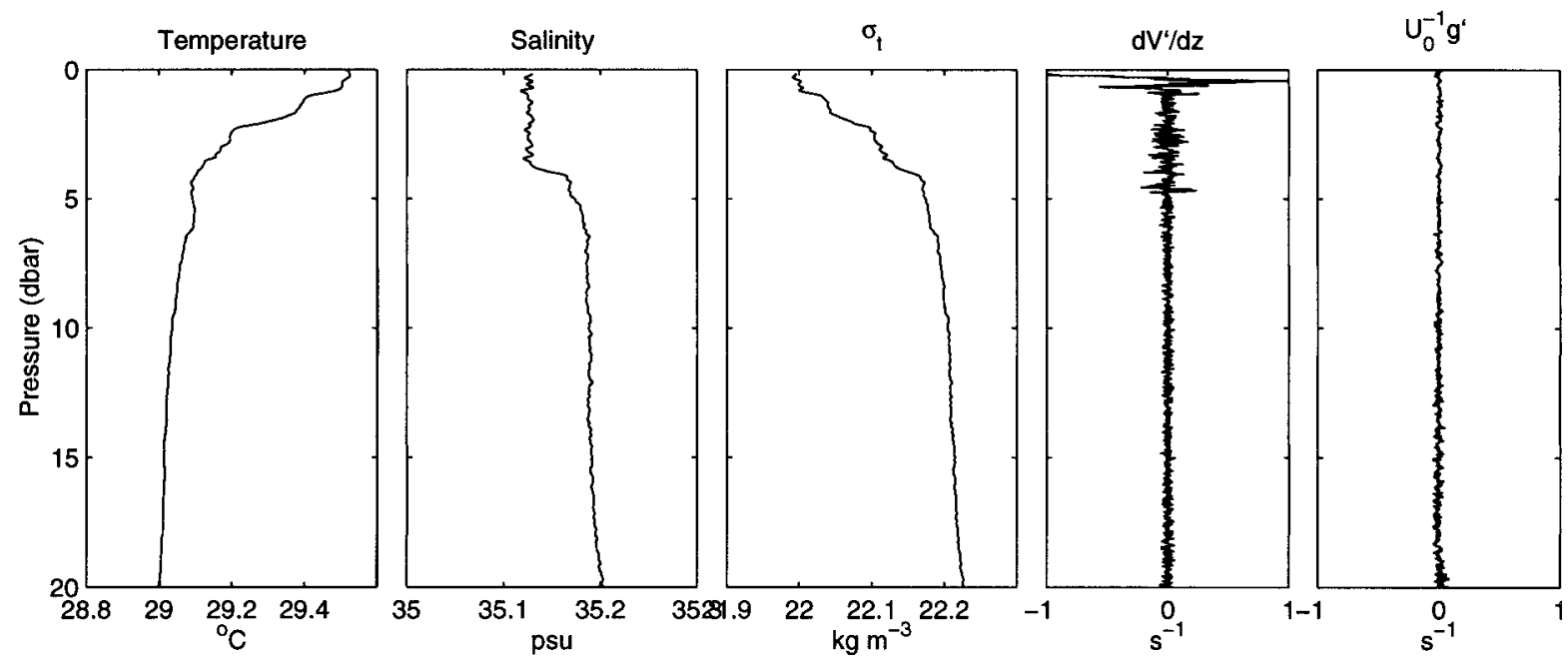

FIG. 7. An example of measurement by free-rising profiler within a shallow freshwater lens in the western equatorial Pacific (0429 UTC 15 Apr $\left.1994 ; 1^{\circ} 58^{\prime} \mathrm{N}, 165^{\circ} 00^{\prime} \mathrm{E}\right)$ under conditions of low wind speed $\left(2.1-3.7 \mathrm{~m} \mathrm{~s}^{-1}\right)$. Note increase of velocity derivative fluctuations $\left(d V^{\prime} / d z\right)$ within the upper $4.5 \mathrm{~m}$. The profiler's own vibrations $\left(U_{0}^{-1} g^{\prime}\right)$ are much less than $d V^{\prime} / d z$, where $U_{0}$ is the vertical velocity of the profiler. Vertical (longitudinal) velocity $\left(V^{\prime}\right)$ and acceleration $\left(g^{\prime}\right)$ signals are high-passed with a 4-Hz cutoff frequency and low-passed with a $40-\mathrm{Hz}$ cutoff frequency.

Note the increase of velocity derivative fluctuations $(d V /$ $d z$ ) in the upper $4.5 \mathrm{~m}$.

\section{Conclusions}

Requirements for the near-surface turbulence sensor system are different from those for the deep ocean measurements. Because of relatively high dissipation rates near the air-sea interface, the requirements on the noise levels of the velocity sensor are not as stringent as for deep ocean measurements. For near-surface measurements, a serious problem is the surface wave disturbances. The surface wave velocity disturbance appears to be about two orders of magnitude larger than the turbulence signal.

For TOGA COARE, a new type of the electromagnetic velocity probe was employed for turbulence measurements in the near-surface layer of the ocean. The use of the external part of the magnetic field improves the hydrodynamic form of the primary sensor and provides a low hydrodynamics noise level for a wide flowspeed range $\left(0-12.5 \mathrm{~m} \mathrm{~s}^{-1}\right)$. The electromagnetic velocity probes were mounted on the bow of the R/V Moana Wave and on a free-rising profiler. The high relative speed of the measurements provided effective separation of the turbulence and surface wave signals. The linearity of output characteristics of the electromagnetic velocity sensor and the localization in narrow frequency bands of the vibrations of the bow sensors allowed calculation of dissipation rate using the Stewart and Grant techniques with estimated accuracy of $\sim 2 \times$ $10^{-9} \mathrm{~W} \mathrm{~kg}^{-1}$. A coherent noise reduction algorithm effectively removes vibration contamination of the longitudinal $\left(V_{x}\right)$ velocity dataset, further improving the accuracy of the dissipation rate estimates to $10^{-11} \mathrm{~W}$ $\mathrm{kg}^{-1}$. Contouring the near-surface layer of the ocean using the depth variation of sensors because of surface waves and associated ship's pitching provides a new opportunity to analyze the near-surface turbulence in its connection to the spatial structure. The free rising profiler provides fine resolution of microstructure and turbulence in the near-surface layer of the ocean.

Acknowledgments. We thank Michael Gregg (University of Washington), who suggested the inclusion of this work to the TOGA COARE IOP planners and provided the opportunity to collect measurements during the R/V Moana Wave COARE IOP-3 leg. Sharon DeCarlo (University of Hawaii) and Dmitry Khlebnikov (P. P. Shirshov Institute of Oceanology) wrote software for recording and preliminary processing of the data. Jefrey Snyder (University of Hawaii) provided technical support of the measurements. The assistance of the crew of the R/V Moana Wave was crucial for success of the program. Yuri Golubev (Granit, St. Petersburg, Russia) provided precruise calibration of the velocity sensors. The P. P. Shirshov Institute of Oceanology rendered substantial assistance on the first stages of the project including providing the ship's time on the R/V Academic Ioffe in 1992 for preliminary field tests. This work has been supported by ONR Grants N00014-961-0832 and N00014-96-1-0836 and NSF Grant OCE-9216891.

\section{APPENDIX A}

\section{Theory of a Three-Component Electromagnetic Velocity Sensor}

Figure A1a shows a schematic diagram of the electromagnetic sensor for three velocity components. The 

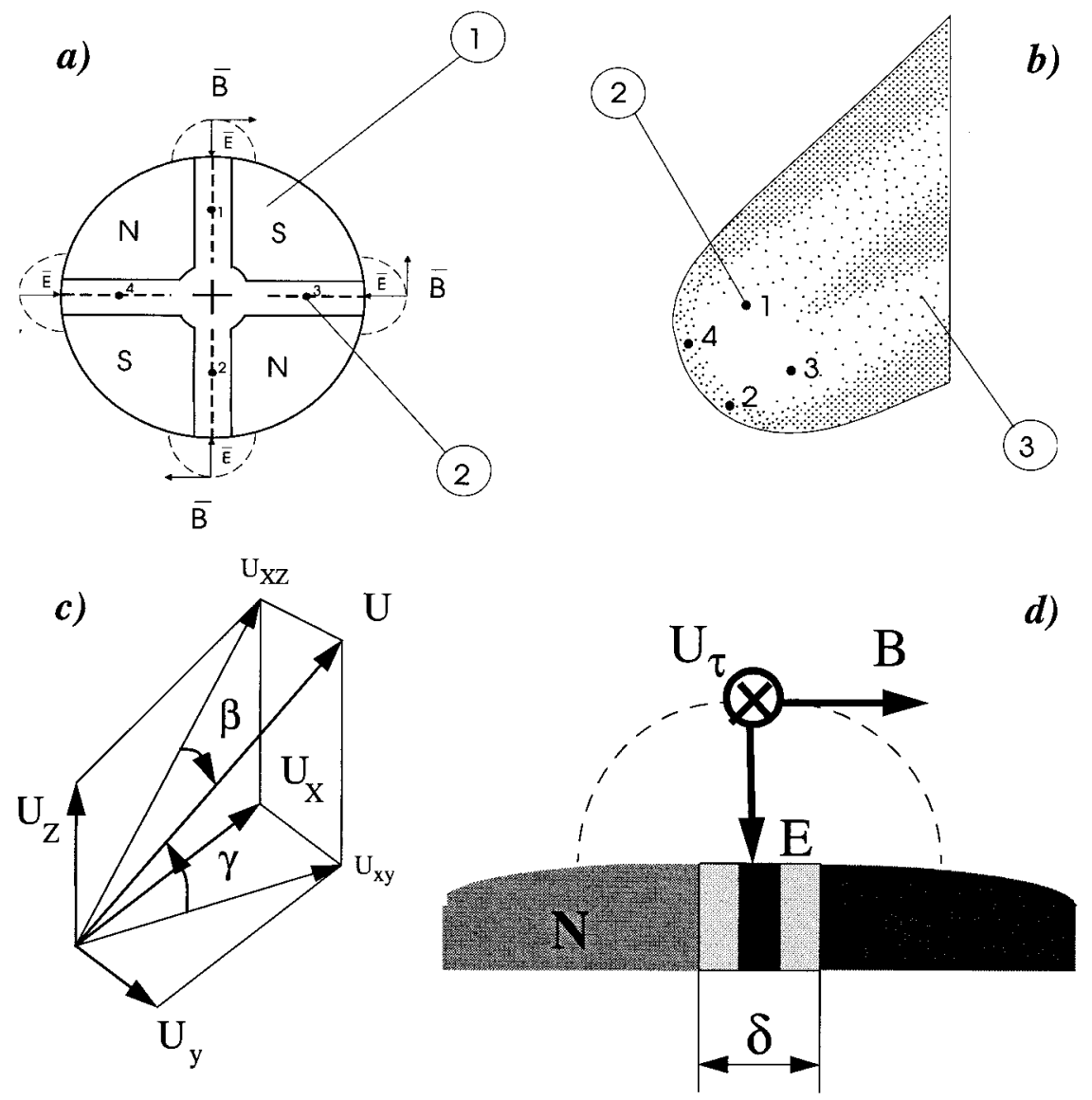

FIG. A1. Schematic diagram of electromagnetic velocity sensor showing the (a), (b) system of magnets and electrodes, (c) coordinate system, and (d) the magnetic and electric field structure in the vicinity of an electrode.

system of permanent magnets and electrodes (Fig. A1a) allows us to measure all three components of the velocity vector. Seawater flowing in the magnetic field $\mathbf{B}$ (see Fig. A1d) generates an electric field E, which obeys Maxwell's equations of electrodynamics (Landau and Lifshitz 1960):

$$
\begin{aligned}
\mathbf{J} & =\sigma\left(\mathbf{E}+\frac{\mathbf{V} \times \mathbf{B}}{c}\right), \\
\operatorname{div} \mathbf{J} & =0,
\end{aligned}
$$

where $\mathbf{J}$ is the current density, $\mathbf{V}$ is the relative velocity vector, and $c$ is the speed of light. In cylindrical coordinates $r, \psi$, and $\tau$,

$$
\operatorname{div} \mathbf{J}=\frac{1}{r} \frac{\partial}{\partial r}\left(r J_{r}\right)+\frac{1}{r^{2}} \frac{\partial J_{\psi}}{\partial \psi}+\frac{\partial J_{\tau}}{\partial \tau} .
$$

For a magnetic field with cylindrical symmetry $\partial J_{\psi} / \partial_{\psi}$ $=0$ and $\partial J_{\tau} / \partial \tau=0$. Equation (A2) therefore reduces to

$$
\frac{1}{r} \frac{\partial}{\partial r}\left(r J_{r}\right)=0
$$

Integration of (A4) from $r_{0}$ to $r$ results in

$$
J_{r}=\frac{J_{0} r_{0}}{r}
$$

where $r_{0}$ is some reference radius (e.g., the radius of electrode) and $J_{0}=J_{r}\left(r_{0}\right)$. The measuring electrode is connected to the input of the low-frequency amplifier with a very large input impedance. We can therefore assume with good accuracy that $J_{0}=0$ and therefore $\mathbf{J}=0$ in Eq. (A1). Correspondingly, Eq. (A1) reduces to

$$
E=\frac{\partial \varphi}{\partial r}=-\frac{V_{\tau} B}{c}
$$

where $\varphi$ is the potential of electric field, $V_{\tau}$ is the tangential component of velocity in the vicinity of electrode, and $B$ is the magnitude of magnetic induction vector. 
The external part of magnetic field can be approximated in the following way:

$$
B \approx B_{0} \exp (-z / \delta),
$$

where $\delta=a l, l$ is the magnetic gap width, and $a$ is the proportionality coefficient. Assuming that $\varphi(\infty)=0$, we obtain by integrating (A6) that

$$
\begin{aligned}
\varphi & =\int_{0}^{\infty} E d r \approx-c^{-1} B_{0} V_{\tau} \int_{r_{0}}^{\infty} \exp (-z / \delta) d z \\
& =A c^{-1} B_{0} V_{\tau},
\end{aligned}
$$

where $A=\exp \left(-r_{0} / \delta\right)$ is the proportionality coefficient depending on configuration of the magnetic system and the measuring electrode diameter. According to (A8), the electric potentials of electrodes are proportional to the corresponding tangential components of velocity field in the vicinity of the electrodes:

$$
\varphi_{i} \sim V_{\tau_{i}},
$$

where $i=1-4$.

According to Streeter (1962), the tangential velocity near the sphere in the uniform stream can be estimated as follows:

$$
V_{\tau}=a U \sin \theta
$$

where $\theta$ is the polar angle in spherical coordinates and $a$ is the coefficient (for sphere, $a=1.5$ ). The stagnation point occurs on the axis when $\theta=0$. When the stream is directed along the longitudinal axis of the sensor, the magnitude of tangential velocity in the vicinity of electrodes is equal to $V_{\tau_{i}}=a U \sin \theta_{0}(i=1-4)$. When the fluctuation of velocity vector occurs in the longitudinal (x) direction, it equally changes the magnitude of the tangential velocity near all four electrodes. When the fluctuation of velocity vector occurs in the transversal $(y, z)$ direction, it changes the position of the stagnation point on the body of the sensor as well; this results in an asymmetrical change of the tangential velocities near the electrodes.

In the general case, the relative fluid velocity (with respect to the right-hand coordinate system connected to the sensor) is specified by the velocity components $V_{x}, V_{y}$, and $V_{z}$. It can also be expressed in terms of an angle of pitch $\gamma$ and an angle of drift or sideslip $\beta$, defined by (Fig. A1)

$$
\gamma=a \sin \frac{V_{z}}{U}, \quad \beta=a \sin \frac{V_{y}}{U},
$$

in which $U^{2}=V_{x}^{2}+V_{y}^{2}+V_{z}^{2}$. Conversely,

$$
\begin{aligned}
& V_{x}=U \cos \gamma \cos \left(a \sin \frac{\sin \beta}{\cos \gamma}\right), \\
& V_{y}=U \sin \beta, \\
& V_{z}=U \sin \gamma .
\end{aligned}
$$
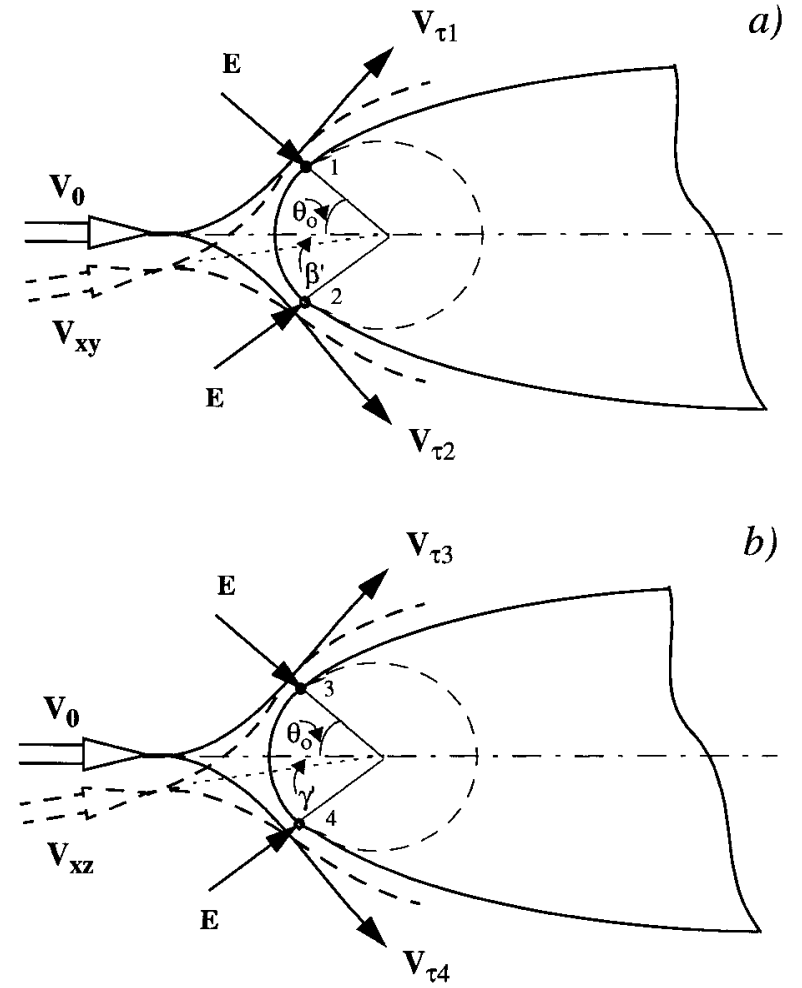

FIG. A2. Decomposition of velocity vector on a half-body of the sensor: (a) plane section $x-y$ passing through symmetry axis $x$ and electrodes 1 and 2 (see Fig. A1) and (b) plane section $x-z$ passing through symmetry axis $x$ and electrodes 3 and 4 (see Fig. A1).

For small velocity fluctuations of the velocity vector in a transversal direction, that is, at

$$
V_{y} \ll U \text { and } V_{z} \ll U,
$$

expression (A12) reduces to

$$
V_{x} \approx U \text {. }
$$

Figure A2 shows decomposition of velocity vector on a half-body of the velocity sensor. Under condition (A15) $\beta \approx-\beta^{\prime}$ and $\gamma \approx \gamma^{\prime}$, where $\beta^{\prime}$ and $\gamma^{\prime}$ are the polar angles of the stagnation point in the corresponding $x-y$ and $x-z$ planes $\left(-\pi<\beta^{\prime}<\pi\right.$ and $-\pi<\gamma^{\prime}<$ $\pi)$. The magnitude of tangential velocity in the vicinity of electrodes $1-4$ can be represented as follows:

$$
\begin{aligned}
& V_{\tau_{1}} \approx a U \sin \left(\frac{\pi}{2}-\theta_{0}-\gamma\right), \\
& V_{\tau_{2}} \approx a U \sin \left(\frac{\pi}{2}-\theta_{0}+\gamma\right), \\
& V_{\tau_{3}} \approx a U \sin \left(\frac{\pi}{2}-\theta_{0}+\beta\right), \quad \text { and } \\
& V_{\tau_{4}} \approx a U \sin \left(\frac{\pi}{2}-\theta_{0}-\beta\right) .
\end{aligned}
$$


According to (A13)-(A20) and from the trigonometrical identities,

$$
\sin \left(\frac{\pi}{2}-\theta_{0}+\beta\right)-\sin \left(\frac{\pi}{2}-\theta_{0}-\beta\right)=2 \sin \beta \sin \theta_{0}
$$

and

$$
\sin \left(\frac{\pi}{2}-\theta_{0}+\gamma\right)-\sin \left(\frac{\pi}{2}-\theta_{0}-\gamma\right)=2 \sin \gamma \sin \theta_{0},
$$

the velocity components of the vector $\mathbf{V}$ can be measured from the combination of electrical potentials from electrodes 1-4:

$$
\begin{aligned}
& V_{y}=U \sin \beta=\frac{\left(V_{\tau_{4}}-V_{\tau_{3}}\right)}{\left(a \cos \theta_{0}\right)}=\frac{\left(\varphi_{3}-\varphi_{4}\right)}{\left(A c^{-1} B_{0} a \cos \theta_{0}\right)}, \\
& V_{z}=U \sin \gamma=\frac{\left(V_{\tau_{2}}-V_{\tau_{1}}\right)}{\left(a \cos \theta_{0}\right)}=\frac{\left(\varphi_{2}-\varphi_{1}\right)}{\left(A c^{-1} B_{0} a \cos \theta_{0}\right)}, \\
& V_{x} \approx U=\frac{\left(V_{\tau_{1}}+V_{\tau_{2}}\right)}{\left(a \cos \theta_{0}\right)}=\frac{\left(\varphi_{1}+\varphi_{2}\right)}{\left(A c^{-1} B_{0} a \cos \theta_{0}\right)}, \text { and } \\
& V_{x} \approx U=\frac{\left(V_{\tau_{3}}+V_{\tau_{4}}\right)}{\left(a \cos \theta_{0}\right)}=\frac{-\left(\varphi_{3}+\varphi_{4}\right)}{\left(A c^{-1} B_{0} a \cos \theta_{0}\right)} .
\end{aligned}
$$

The longitudinal component, $V_{x}$, is measured independently from the two pairs of electrodes [(A23)(A24)]. To double the useful output signal, thus reducing the noise level of the sensor, signals from both pairs of electrodes are being used:

$$
V_{x}=\frac{\left[\left(\varphi_{1}+\varphi_{2}\right)-\left(\varphi_{3}+\varphi_{4}\right)\right]}{\left(2 A c^{-1} B_{0} a \cos \theta_{0}\right)} .
$$

To reduce externally picked-up signals, the differential amplifiers are used. Formula (A23) can therefore be rewritten as follows:

$$
V_{x}=\frac{\left[\left(\varphi_{1}-\varphi_{3}\right)-\left(\varphi_{4}-\varphi_{2}\right)\right]}{\left(2 A c^{-1} B_{0} a \cos \theta_{0}\right)} .
$$

\section{APPENDIX B}

\section{Motion Compensation of Velocity Measurements Using Coherent Noise Cancellation}

The bow sensors measure water velocity relative to the sensor and therefore are susceptible to motion contamination by both ship's motion (primarily low-frequency heave) and vibration at higher frequencies. In fact, the bow velocity measurements made at full ship speed are severely corrupted by sensor motion in several bands, some of which fall into the turbulent microstructure wavenumber bands, as evidenced by Fig. 3d. Note the strong peaks at approximately $18,25,50$, and 110 $\mathrm{Hz}$ in both spectra, and the agreement of the two spectra with respect to the frequency and bandwidth of these peaks. It is evident that almost all the energy in the peaks at 25 and $50 \mathrm{~Hz}$ is a direct result of vibrations.
Further evidence on the nature and degree of contamination can be seen in a plot of the coherence between the two channels, as shown in Fig. 3e. The contamination centered around $0.4 \mathrm{~Hz}$ is associated with ship's motion and is out of band for the microstructure measurements we wish to make. Above $10 \mathrm{~Hz}$ there is a varying degree of contamination, with high coherence at $18,25,50$, and $110 \mathrm{~Hz}$, and possibly somewhat below $10 \mathrm{~Hz}$. Ad hoc procedures for motion compensation, such as extrapolating the spectrum through known motion peaks or using a notch filter, are relatively ineffective here because the resonant properties of the bow frame depend on the position of the air-water interface with respect to the frame, which changes during the pitching period.

The high coherence between the measured water velocity and sensor package motion (integrated accelerometer), however, demonstrates that these two time series are related by a time-invariant linear transfer function and suggests that the accelerometer measurements can be used to attenuate the motion contamination in the velocity measurements in an optimal fashion using coherent noise cancellation techniques.

\section{a. Coherent noise cancellation}

The objectives of the analysis presented in this section are to develop and demonstrate effective motion compensation for velocity data based on coherent noise reduction (Schoeberlein and Baker 1996). Coherent noise cancellation techniques have four advantages for decontamination of velocity measurements. First, using the coherence between the integrated accelerometer and velocity sensor permits that fraction of the measured velocity due to sensor motion to be eliminated without modifying the underlying ocean spectrum. Variance at frequencies associated with sensor motion is not merely eliminated, as, for example, by notch filtering. Only that fraction of the total measured signal that is coherent with the sensor velocity is removed. Second, these techniques are flexible in that they can be applied in both the frequency (or wavenumber) domain or the temporal (or spatial) domain. Third, they are optimal in the least squares sense by removing velocity variance that is coherent with sensor motion by minimizing the output variance. They are completely insensitive to unknown phase or gain characteristics; the weights of the filter are varied to minimize the output variance and can thus compensate for lack of detailed knowledge of the system transfer functions. Finally, such techniques can be fixed or adapt to slowly changing noise environments.

To demonstrate the effectiveness of this technique, we will examine a sample of velocity and acceleration data from TOGA COARE in which all sensors were in the water continuously, implement the optimal Weiner filter in the time domain to noise cancel the data, and compare the spectra and coherence between the velocity and sensor motion before and after noise cancellation. 


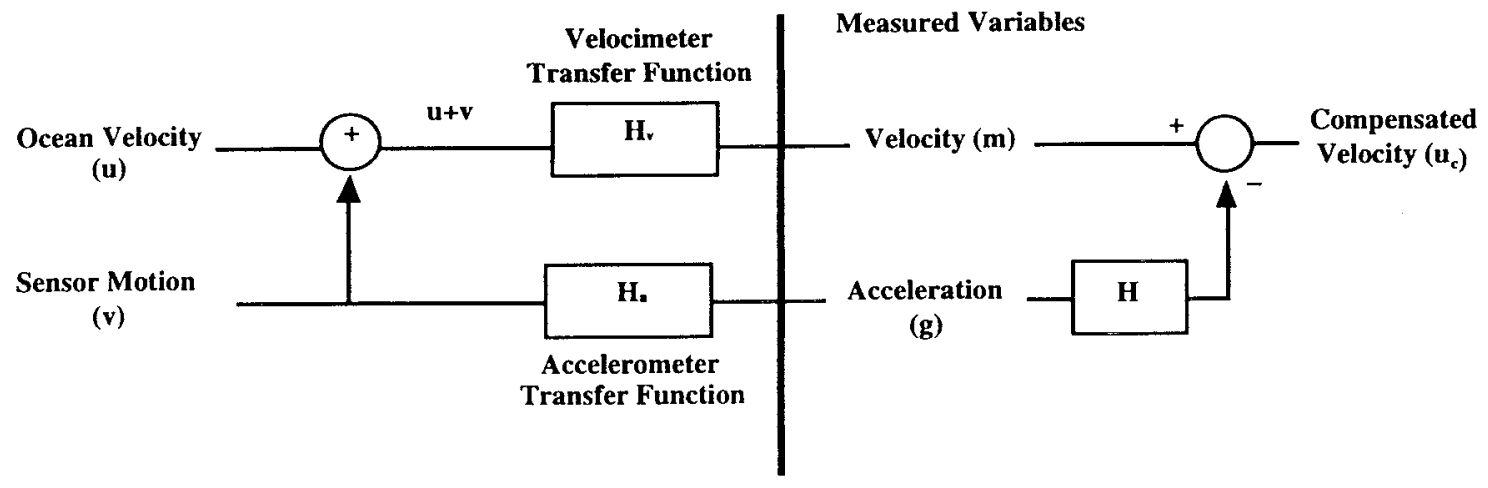

$$
\begin{aligned}
\gamma_{g m}^{2}(\omega)=\frac{\left|P_{g m}(\omega)\right|^{2}}{P_{m m}(\omega) P_{g g}(\omega)} & \text { If } H(\omega)=\frac{P_{g m}(\omega)}{P_{g g}(\omega)}=\frac{H_{v}(\omega) H_{a}^{*}(\omega)}{\left|H_{a}(\omega)\right|^{2}} \\
=\frac{P_{v \nu}(\omega)}{P_{u \nu}(\omega)+P_{v \nu}(\omega)} & \text { Then } \\
\text { Assumes } \quad \gamma_{u \nu}^{2}(\omega)=0 &
\end{aligned}
$$

FIG. B1. Measurement scenario and compensation for motion contamination. The measured velocity is the sum of the ocean velocity and the sensor motion as seen through the transfer function of the velocity sensor. The sensor motion, $v$, is also measured through its transfer function, which effectively includes differentiation into acceleration $(g)$. After the measurement is made, if the composite transfer function $(H)$ can be estimated from the data, or known sensor response characteristics, then the motion can be removed. Imperfect knowledge of the transfer functions and offsets between the velocity sensors and accelerometers makes direct estimation of the required transfer function difficult.

Figure B1 shows a linear model of the velocity contamination process. With detailed knowledge of the system transfer function of the velocimeter and accelerometer, the velocity measurements can be decontaminated by properly filtering the accelerometer data and subtracting it from the measured velocity data. This approach to motion removal not only assumes perfect knowledge of these transfer functions but also assumes that the motion of the accelerometer is the same as that of the velocity sensor, which is a poor assumption in that these sensors are not colocated in the bow sensor suite.

\section{b. Coherent noise cancellation of velocity measurements}

The approach taken here is to filter the integrated acceleration using a simple Weiner filter, $W(t)$, and subtract it from the measured velocity, as shown in the upper panel of Fig. B2. The form of the Wiener filter is shown in the lower panel of Fig. B2. It consists basically of $N$ one-sample lags (or taps) that are multiplied by the same number of weights and summed. The issue is how to estimate the weights required for good noise cancellation.

The Weiner filter is fully discussed in Widrow and Sterns (1985), as well as elsewhere. The filter weights are chosen to minimize the mean square error, which, in this application, is the variance of the motion compensated water velocity. Note that the variance is not set to zero and only that fraction of the variance of the water velocity at frequencies coherent with the motion of the sensor package is removed. The solution for the optimal weight vector is straightforward and given by the following equations:

$$
\mathbf{R}=\left\langle\mathbf{X} \cdot \mathbf{X}^{\mathrm{T}}\right\rangle, \quad \mathbf{P}=\left\langle\mathbf{y} \cdot \mathbf{X}^{\mathrm{T}}\right\rangle, \quad \text { and } \quad \mathbf{W}=\mathbf{R}^{-1} \mathbf{P},
$$

where $\mathbf{X}$ is called the reference input (the integrated accelerometer data), $\mathbf{y}$ is the primary input (the measured velocity data), $\mathbf{W}$ is the optimal weight vector, and \langle\rangle indicates averaging over all measurements. For $N$ weights, $\mathbf{R}$ is the $N \times N$ reference correlation matrix, and $\mathbf{P}$ is the $1 \times N$ cross-correlation vector. One important aspect regarding the implementation of the filter is to ensure that the reference correlation matrix is not singular and thus can be inverted. This can be a problem when using data sampled at a high rate (such as 400 $\mathrm{Hz}$ ) that contains a strong low-frequency component, such as the ship's motion in the accelerometer measurements at $0.4 \mathrm{~Hz}$. The correlation function is nearly constant over the 30-60 lags (0.075-0.15 s), making the correlation matrix nearly singular. We have chosen not to noise cancel this component of the motion contamination because it is out of band for calculation of the kinetic energy dissipation rate. Therefore, both the velocity and integrated accelerometer data presented here have been high-pass filtered at $10 \mathrm{~Hz}$ by subtracting the original data from a running average over $0.1 \mathrm{~s}$.

Figure B3 presents the spectra of the high-passed data. These results represent the data prior to noise can- 

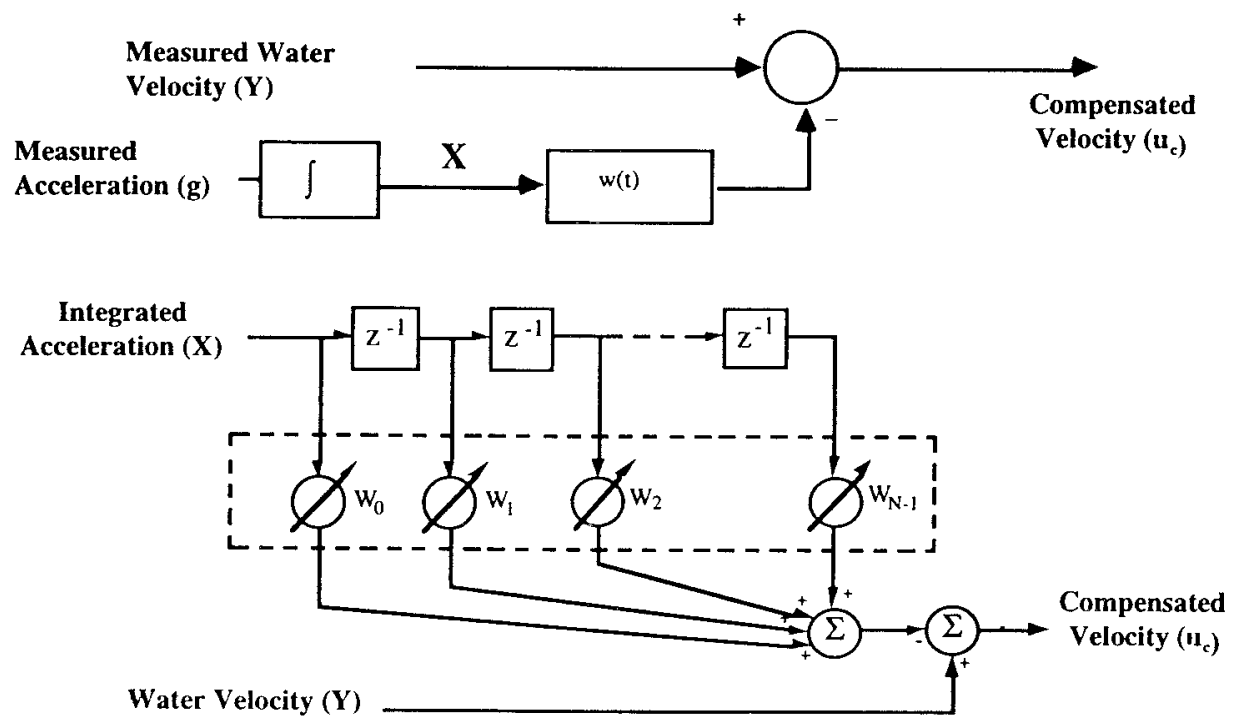

FIG. B2. (top) General motion compensation flow. (bottom) Filter structure used in the Weiner filter.

cellation and will be compared to the noise canceled results after using 1) a simple scaled subtraction, 2) a Weiner filter with 13 weights, and 3) a Weiner filter with 60 weights. The effectiveness of noise cancellation is assessed by examining the reduction in the spectral levels associated with motion and reduction of the coherence levels. The spectra show strong contamination at 25 and $50 \mathrm{~Hz}$, as well as lesser contamination at 8, 15, 110 , and $150 \mathrm{~Hz}$.

In Fig. B4, the velocity signal has been noise canceled using a simple scaled difference, which is similar to a one-weight Weiner filter. The weight used to scale the integrated accelerometer prior to subtraction from the velocity was determined by least squares to be about 1.4. Although the motion peaks in the velocity have been attenuated, there is still ample evidence of their presence at 25, 50, and $110 \mathrm{~Hz}$. Motion contamination is noticeable in the spectrum, and obvious in the co-

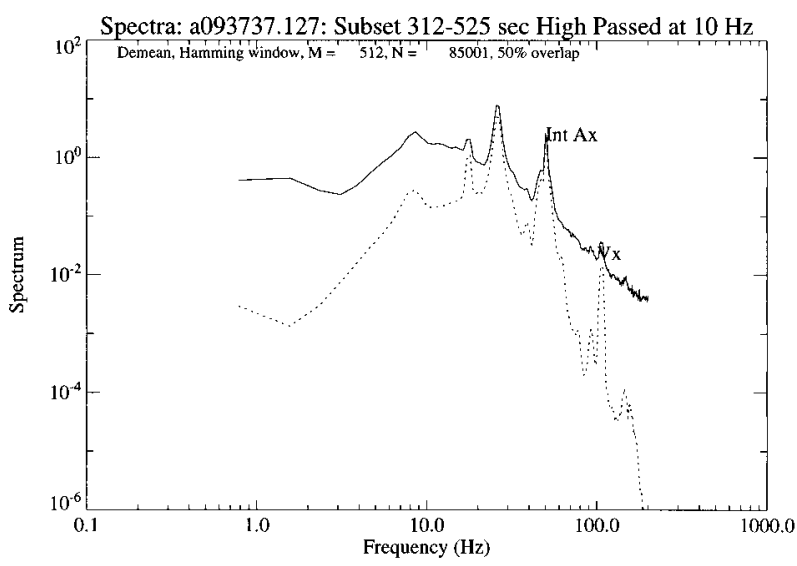

FIG. B3. Spectra of measured water velocity and integrated acceleration prior to corresponding noise cancellation. herence, where the broad plateau from 3 to $70 \mathrm{~Hz}$ is gone, but there is still statistically significant coherence at peaks within this range.

Figure B5 presents results for the Weiner filter with 13 weights. The spectra now show very little evidence of any contamination at the vibration frequencies, and the coherence also indicates very little residual motion contamination remaining in the output time series. There are a few small peaks left in the coherence (at 8, 25, and $150 \mathrm{~Hz}$ ), whose $95 \%$ confidence intervals do not enclose zero coherence. Increasing the number of weights to 60, as presented in Fig. B6, improves the results. The velocity spectrum appears free of motion contamination as before, but now the $95 \%$ confidence interval for the coherence encloses zero throughout the frequency band from 1 to $200 \mathrm{~Hz}$.

\section{c. Modifications for estimation of dissipation rate}

Calculation of the dissipation rate proceeds from the spatial derivatives of the measured velocity, which is proportional to the temporal derivative of velocity. One of the distinct advantages of estimating the optimal transfer function to remove motion contamination is that the motion compensation can be carried out using either the velocity or velocity derivatives. That is, the velocity can be compensated using integrated acceleration as an estimate of sensor motion, or the velocity can be differentiated and noise canceled using the raw acceleration. Although in these two cases the Weiner filter coefficients will be significantly different, both filters will be optimal for each case, and the degree of noise cancellation is determined only by the coherence between the reference and primary channels, which is unaffected by the introduction of any linear, time-invariant operation between the channels. 

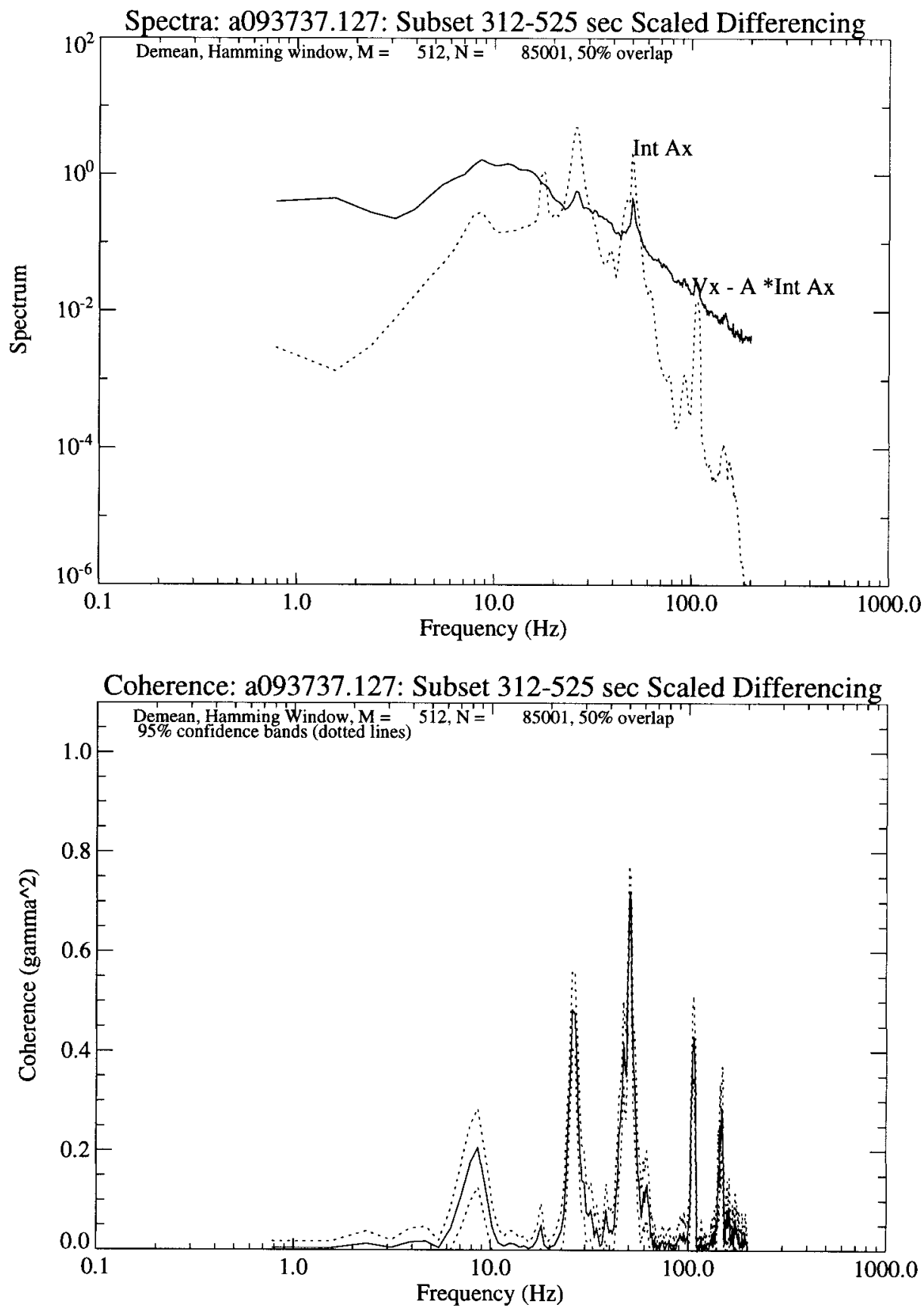

FIG. B4. (a) Spectra and (b) coherence of measured water velocity and integrated acceleration after noise cancellation using a simple scaled difference.

To illustrate this, we implemented motion compensation for the velocity derivative, as illustrated in Fig. B7. The acceleration is used as the reference channel, and the differentiated velocity is the primary input. One advantage of basing the motion compensation on acceleration and differentiated velocity is that the additional step of high passing the data is no longer required. Because there is relatively little variance at low frequencies in the acceleration data, the reference corre- lation matrix is further from singularity than in the nondifferentiated case, and thus does not require high-pass filtering. The velocity is differentiated using the following four-point filter, $y_{i}=\left[x_{i-3}-x_{i}+27\left(x_{i-1}-x_{i+1}\right)\right] /$ $(24 \Delta t)$, where $y_{i}$ is the differentiated output, $x_{i}$ is the input, and $\Delta t$ is the time between samples. We will consider the transfer function of this differentiator in a moment. Figure B8a presents spectra of the acceleration and differentiated velocity in the $x$ direction for the same 

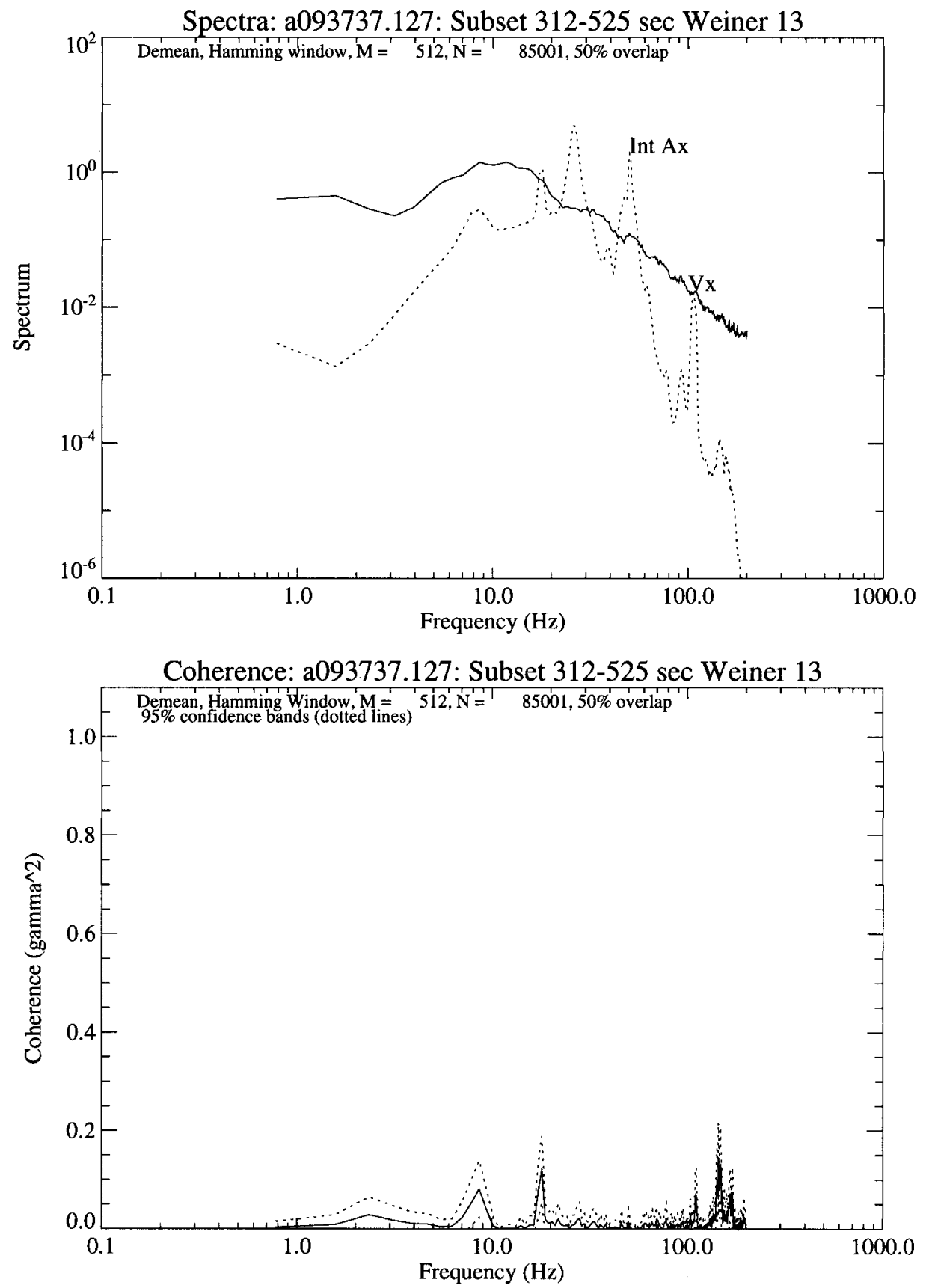

FIG. B5. (a) Spectra and (b) coherence of measured water velocity and integrated acceleration after noise cancellation using a Weiner filter with 13 weights.

time period as that discussed previously. Figure B8b is the corresponding coherence. Figure B9a presents the acceleration and differentiated velocity after motion compensation using a 60-weight Weiner filter, and Fig. B9b presents the residual coherence. As before, the $95 \%$ confidence intervals of the coherence enclose zero, just as in Fig. B6b for the velocity and integrated acceleration.

When estimating the dissipation rate from short spec- tra of the differentiated, motion-compensated velocity measurement, we must take into account the transfer function of the differentiator and provide a correction in frequency space that can be applied to the spectra. The magnitude of the transfer function corresponding to this differentiator is

$$
|H(\omega)|^{2}=\frac{[\sin (3 \omega \Delta t)-27 \sin (\omega \Delta t)]}{\left(144 \Delta t^{2}\right)} .
$$


Spectra: a093737.127: Subset 312-525 sec Weiner 60

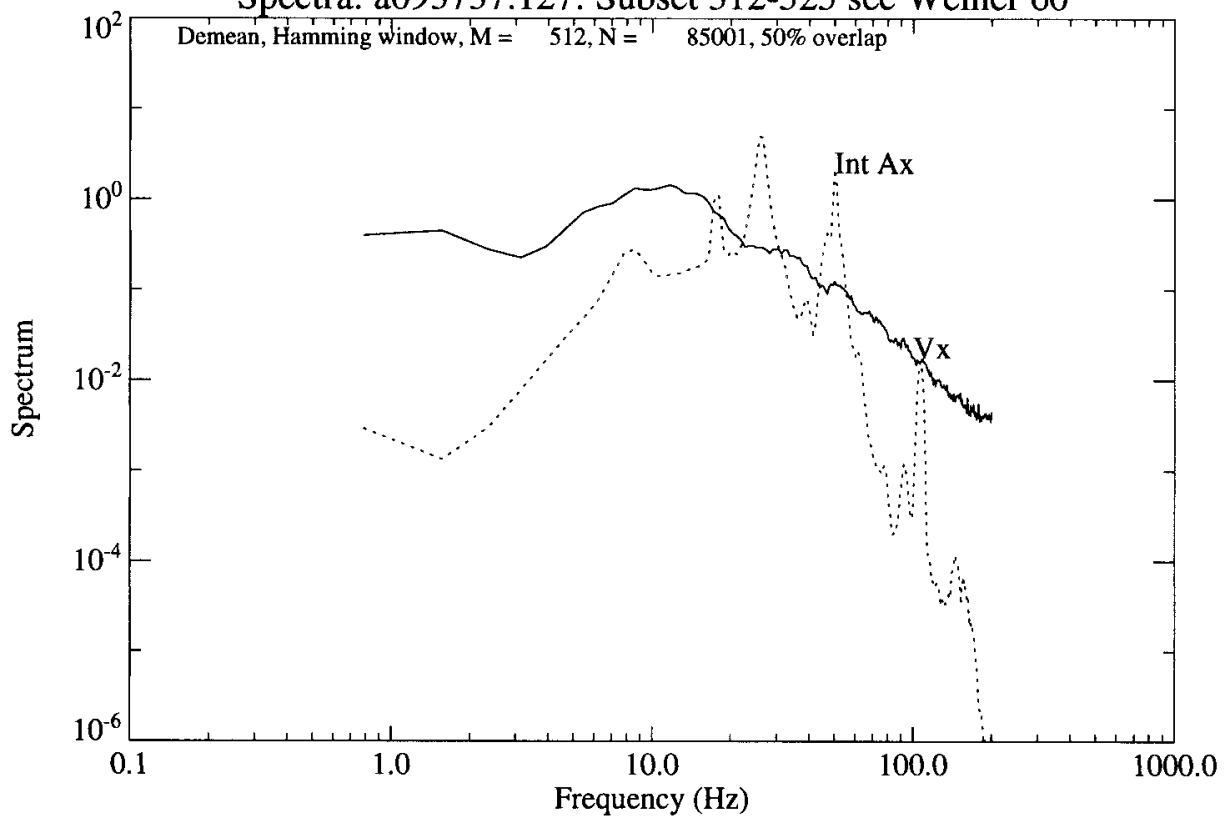

Coherence: a093737.127: Subset 312-525 sec Weiner 60

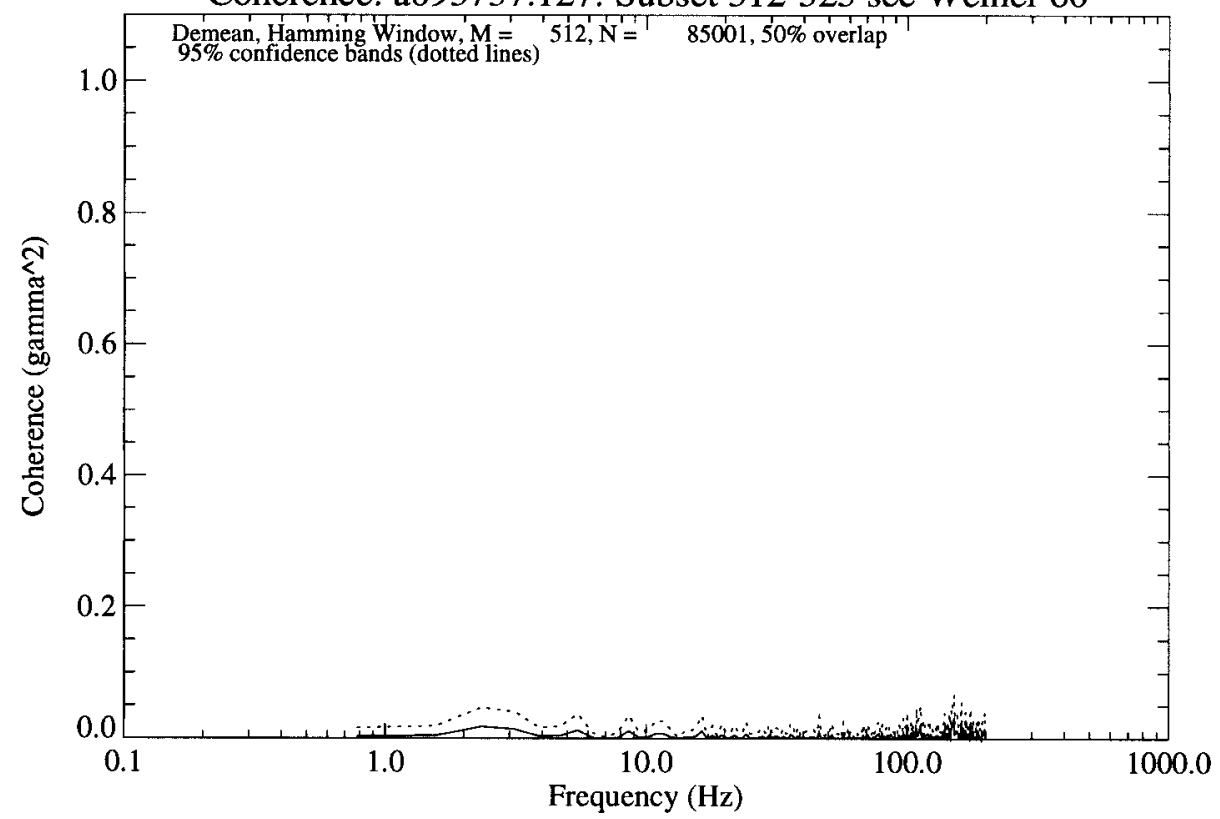

FIG. B6. (a) Spectra and (b) coherence of measured water velocity and integrated acceleration after noise cancellation using a Weiner filter with 60 weights.

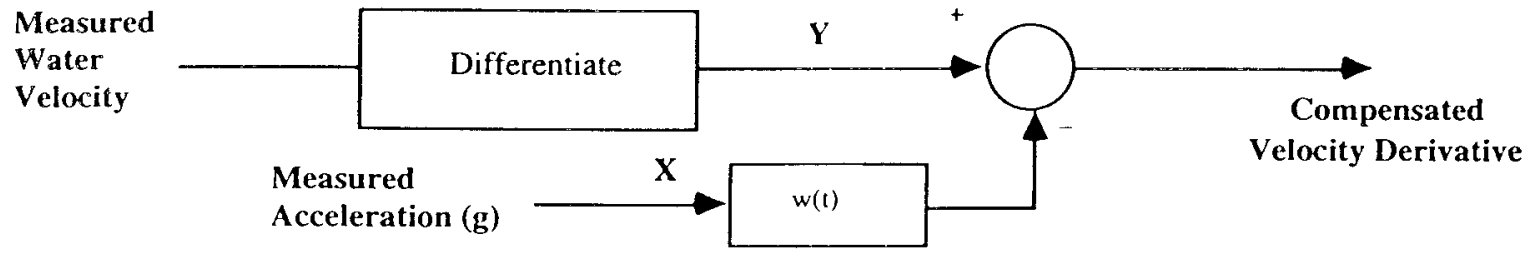

FIG. B7. Flowchart of motion compensation for velocity derivative. 

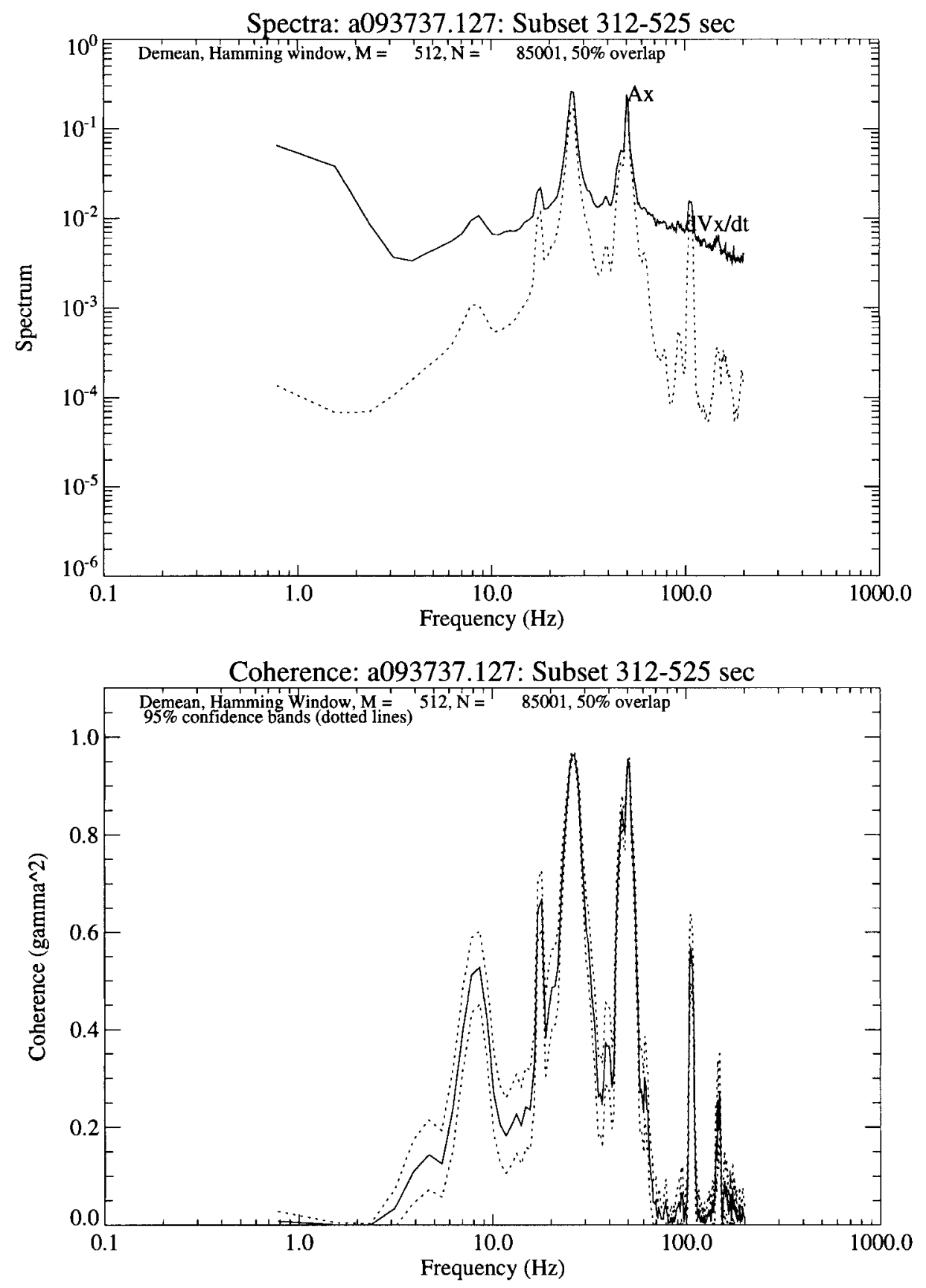

FIG. B8. (a) Spectra and (b) coherence of acceleration and differentiated velocity in $x$ direction.

\section{d. Effects on dissipation rate estimates}

The velocity sensor does not resolve the full velocity spectrum. A power-law method, based on the $k^{-5 / 3}$ (inertial-viscous subrange) region of the universal velocity spectrum, is discussed in the main body of this paper. This method builds on the approach of Grant and Stewart (1962) and is an iterative-based fit of the inertialviscous subrange to the empirical spectrum that excludes the vibration-induced noise. This method should work well given a well-resolved spectrum (long FFTs and data records).

An alternative method, appropriate only for noisecanceled data, is based on comparing the measured velocity gradient variance within a specified wavenumber band with the variance that would be estimated from the universal spectrum. Using noise-canceled velocity data, this method allows accurate estimates over short segments. 

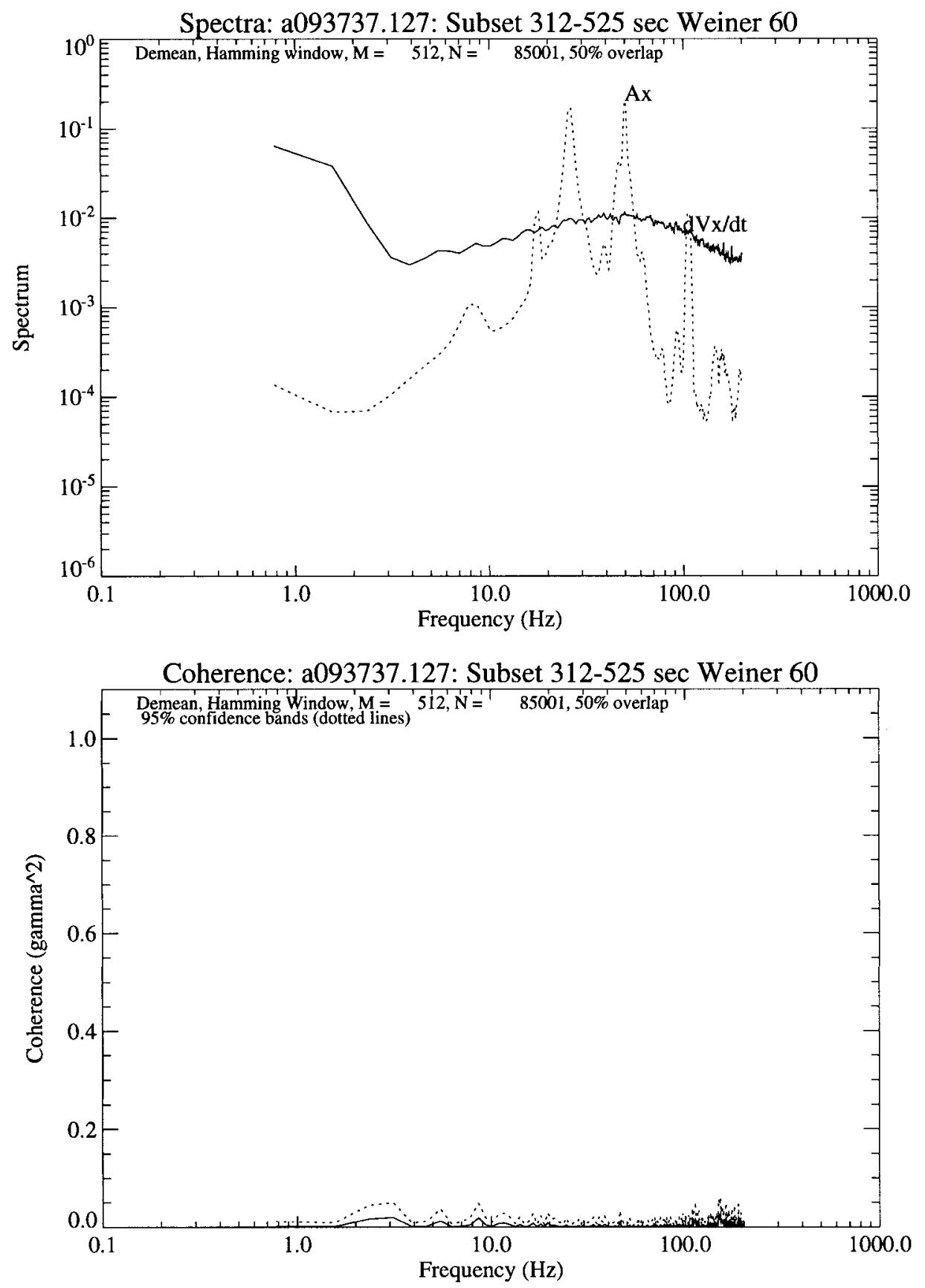

FIG. B9. (a) Spectra and (b) coherence of differentiated water velocity and acceleration after noise cancellation using a 60-weight Weiner filter.

For the second method, the expression for the universal one-dimensional spectrum is solved for $\varepsilon$ and yields $\varepsilon=\left\{\alpha \sigma^{-2}\left[k_{1}^{4 / 3}-k_{0}^{4 / 3}\right]\right\}^{-3 / 2}$, where $\alpha=0.75(18 /$ $55)(1.44)$ and $\sigma^{2}$ is the velocity gradient variance derived from the wavenumber band defined by $k_{1}$ and $k_{0}$.

An example of the dissipation rates estimated by this method for the data discussed in this appendix is shown in Fig. B10 along with the result one would obtain by naively applying this method to the raw data. The es- timates were based on spectra from 5-m segments, a bandwidth of $0.5-30 \mathrm{cpm}(2.7-160 \mathrm{~Hz})$. Corrections for the antialias filter, sensor response, and the differentiator have been applied to each spectrum. Figure B10a compares the dissipation rates from the measured velocity $\left(V_{x}^{\prime}\right)$ with estimates derived from the accelerometer. The results indicate significant contributions to the dissipation rate from motion contamination of the raw velocity gradient. As expected from the coherence results 

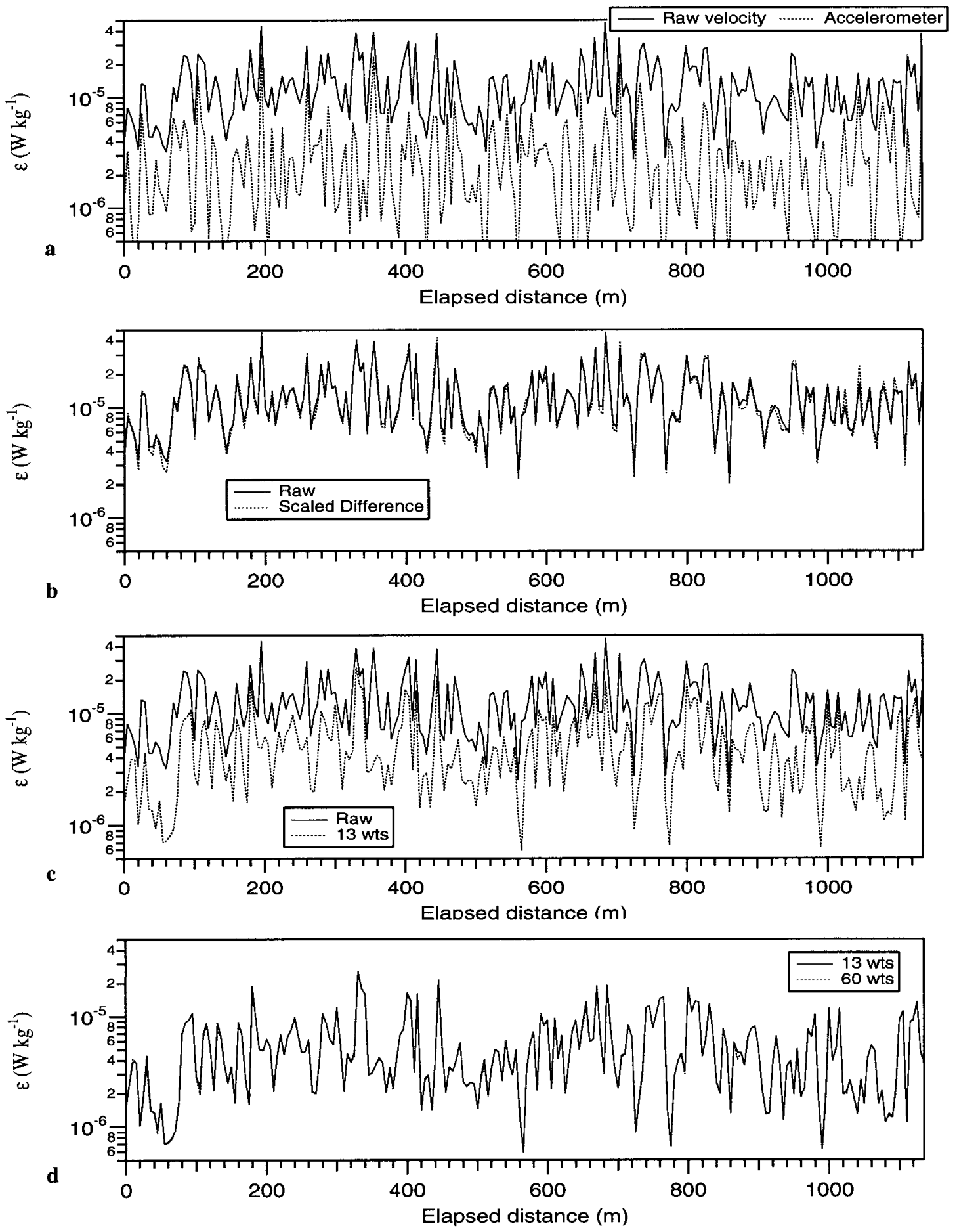

FIG. B10. Comparison of dissipation rates for data segment 093737.127 derived from (a) raw velocity vs acceleration, (b) raw velocity vs a simple scaled difference time series, (c) scaled difference vs 13-weight noise-cancelled time series, and (d) 13 - vs 60 -weight noise-cancelled time series.

presented above, $\varepsilon$ estimates based on the simple scaled difference show virtually no improvement over results from uncompensated velocity gradient. The results derived from the noise-canceled time series using 13 weights show significant reduction in the values of $\varepsilon$, thereby explicitly demonstrating that a large fraction of the kinetic energy dissipation rate in uncorrected $\varepsilon$ estimates is merely sensor vibration. This represents a 
significant improvement in the accuracy of the $\varepsilon$ estimates when compared with the raw data estimates. Slight differences can be observed between the 13- and 60-weight noise-canceled results.

We conclude that coherent noise cancellation techniques can be extremely effective for removing motion contamination of the bow velocity measurements in TOGA COARE using 30-60 weights. The techniques are simple to implement in the time domain, producing uncontaminated velocity estimates for calculations of the dissipation rate.

\section{REFERENCES}

Agrawal, Y. C., E. A. Terray, M. A. Donelan, P. A. Hwang, A. J Williams III, W. M. Drennan, K. K. Kahma, and S. A. Kitaigorodskii, 1992: Enhanced dissipation of kinetic energy beneath surface waves. Nature, 359, 219-220.

Anis, A., and J. N. Moum, 1995: Surface wave-turbulence interactions: Scaling $\varepsilon(z)$ near the sea surface. J. Phys. Oceanogr., 25, 2025-2045.

Arjannikov, A. V., Yu. E. Golubev, and V. A. Zvickevich, 1979: Apparatus for velocity measurement in the water flow (in Russian). USSR patent N679878. Bulletin of Inventions, N30, 1022.

Bye, J. A. T., 1988: The coupling of wave drift and wind velocity profiles. J. Mar. Res., 46, 457-472.

Charnock, H., 1955: Wind stress on a water surface. Quart. J. Roy. Meteor. Soc., 81, 639-640.

Craig, P. D., and M. L. Banner, 1994: Modeling wave-enhanced turbulence in the ocean surface layer. J. Phys. Oceanogr., 24, 25462559.

Fairall, C., E. F. Bradley, J. B. Edson, and G. S. Young, 1996: The TOGA COARE bulk flux algorithm. J. Geophys. Res., 101, 3747-3764.
Landau, L. D., and E. M. Lifshitz, 1960: Electrodynamics of Continuous Media. Addison-Wesley, $417 \mathrm{pp}$.

Mellor, G. L., and T. Yamada, 1982: Development of a turbulent closure model for geophysical fluid problems. Rev. Geophys. Space Phys., 20, 851-875.

Oakey, N. S., and J. A. Elliott, 1982: Dissipation within surface mixed layer. J. Phys. Oceanogr., 12, 175-195.

Schoeberlein, H. C., and M. A. Baker, 1996: Status report: Reduction of motion contamination in TOGA COARE velocity measurements. Johns Hopkins University Tech. Rep. STF-96-104, 20 pp. [Available from Applied Physics Laboratory, The Johns Hopkins University, Laurel, MD 20723.]

Smyth, W. D., P. O. Zavialov, and J. N. Moum, 1997: Decay of turbulence in the upper ocean following sudden isolation from surface forcing. J. Phys. Oceanogr., 27, 810-822.

Soloviev, A., and R. Lukas, 1996: Observation of spatial variability of diurnal thermocline and rain-formed halocline in the western Pacific warm pool. J. Phys. Oceanogr., 26, 2529-2538.

— - N. V. Vershinsky, and V. A. Bezverchnii, 1988: Small-scale turbulence measurements in the thin surface layer of the ocean. Deep-Sea Res., 35, 1859-1874.

_ R. Rukas, S. DeCarlo, J. Snyder, A. Arjannikov, M. Baker, and D. Khlebnikov, 1995: Small-scale measurements near the oceanair interface during TOGA COARE. Data Report, SOEST 9505, University of Hawaii, Honolulu, HI, 257 pp. [Available from Department of Oceanography, University of Hawaii at Manoa, 1000 Pope Rd., Honolulu, HI 96822.]

, - _ - - V. Turenko, M. Baker, and D. Khlebnikov, 1998: A near-surface microstructure sensor system used during TOGA COARE. Part I: Bow measurements. J. Atmos. Oceanic Technol., 15, 563-578.

Stewart, R. W., and H. L. Grant, 1962: Determination of the rate of dissipation of turbulent energy near the sea surface in the presence of waves. J. Geophys. Res., 67, 3177-3180.

Streeter, V. L., 1961: Ideal-fluid flow. Handbook of Fluid Dynamics, McGraw-Hill, 1-73.

Turenko, V. V., 1985: Conductometer (in Russian). USSR patent N1223115.

Widrow, B., and S. D. Sterns, 1985: Adaptive Signal Processing. Prentice-Hall, $474 \mathrm{pp}$ 
Copyright of Journal of Atmospheric \& Oceanic Technology is the property of American Meteorological Society and its content may not be copied or emailed to multiple sites or posted to a listserv without the copyright holder's express written permission. However, users may print, download, or email articles for individual use. 